\title{
Effects of Construction and Operation of a Satellite Power System Upon the Magnetosphere
}

\author{
Prepared by \\ Y. T. CHIU, J. G. LUHMANN, and M. SCHULZ \\ Space Sciences Laboratory \\ The Aerospace Corporation \\ and \\ J. M. CORNWALL \\ Department of Physics \\ University of California, Los Angeles
}

1 December 1979

Prepared for

ARGONNE NATIONAL LABORATORY

Argonne, Illinois 60439

Contract No. 31-109-38-5075 


\section{DISCLAIMER}

This report was prepared as an account of work sponsored by an agency of the United States Government. Neither the United States Government nor any agency Thereof, nor any of their employees, makes any warranty, express or implied, or assumes any legal liability or responsibility for the accuracy, completeness, or usefulness of any information, apparatus, product, or process disclosed, or represents that its use would not infringe privately owned rights. Reference herein to any specific commercial product, process, or service by trade name, trademark, manufacturer, or otherwise does not necessarily constitute or imply its endorsement, recommendation, or favoring by the United States Government or any agency thereof. The views and opinions of authors expressed herein do not necessarily state or reflect those of the United States Government or any agency thereof. 


\section{DISCLAIMER}

Portions of this document may be illegible in electronic image products. Images are produced from the best available original document. 


\section{LABORATORY OPERATIONS}

The Laboratory Operations of The Aerospace Corporation is conducting experimental and theoretical investigations necessary for the evaluation and application of scientific advances to new military concepts and systems. Versatility and flexibility have been developed to a high degree by the laboratory personnel in dealing with the many problems encountered in the nation's rapidly developing space and missile systems. Expertise in the latest scientific developments is vital to the accomplishment of tasks related to these problems. The laboratories that contribute to this research are:

Aerophysics Laboratory: Launch and reentry aerodynamics, heat transfer, reentry physics, chemical kinetics, structural mechanics, flight dynamics, atmospheric pollution, and high-power gas lasers.

Chemistry and Physics Laboratory: Atmospheric reactions and atmospheric optics, chemical reactions in polluted atmospheres, chemical reactions of excited species in rocket plumes, chemical thermodynamics, plasma and laser-induced reactions, laser chemistry, propulsion chemistry, space vacuum and radiation effects on materials, lubrication and surface phenomena, photosensitive materials and sensors, high precision laser ranging, and the application of physics and chemistry to problems of law enforcement and biomedicine.

Electronics Research Laboratory: Electromagnetic theory, devices, and propagation phenomena, including plasma electromagnetics; quantum electronics, lasers, and electro-optics; communication sciences, applied electronics, semiconducting, superconducting, and crystal device physics, optical and acoustical imaging; atmospheric pollution; millimeter wave and far-infrared technology.

Materials Sciences Laboratory: Development of new materials; metal matrix composites and new forms of carbon; test and evaluation of graphite and ceramics in reentry; spacecraft materials and electronic components in nuclear weapons environment; application of fracture mechanics to stress corrosion and fatigue-induced fractures in structural metals.

Space Sciences Laboratory: Atmospheric and ionospheric physics, radiation from the atmosphere, density and composition of the atmosphere, aurorae and airglow; magnetospheric physics, cosmic rays, generation and propagation of plasma waves in the magnetosphere; solar physics, studies of solar magnetic fields; space astronomy, x-ray astronomy; the effects of nuclear explosions, magnetic storms, and solar activity on the earth's atmosphere, ionosphere, and magnetosphere; the effects of optical, electromagnetic, and particulate radiations in space on space systems.

THE AEROSPACE CORPORATION

El Segundo, California 


\section{EFFECTS OF CONSTRUCTION AND OPERATION \\ OF A SA TELLITE POWER SYSTEM \\ UPON THE MAGNETOSPHERE}

$$
\text { Prepared by }
$$

Y. T. Chiu, J. G. Luhmann, and M. Schulz

Space Sciences Laboratory

and

J. M. Cornwall

Department of Physics

University of California, Los Angeles

1 December 1979

Laboratory Operations

THE AEROSPACE CORPORATION

El Segundo, Calif. 90245

Prepared for

ARGONNE NATIONAL LABORATORY

Argonne, Illinois 60439

Contract No. $31-104-38-5075$ 


\section{THIS PAGE \\ WAS INTENTIONALLY \\ LEFT BLANK}

$:$ 
EFFECTS OF CONSTRUCTION AND OPERATION

OF A SATELLITE POWER SYSTEM

UPON THE MAGNETOSPHERE

Prepared

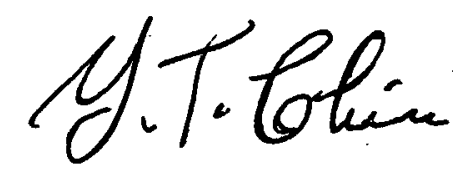

Y. T. Chiu

Ganet Luhmarn

J. G. Luhmann

Approved

\section{Fied de Mare}

F.'A. Morse, Head

Atmospheric Sciences Department

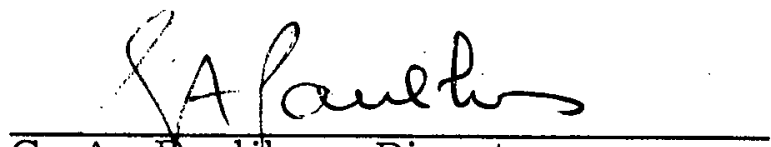

G. A. Paulikas, Director

Space Sciences Laboratory

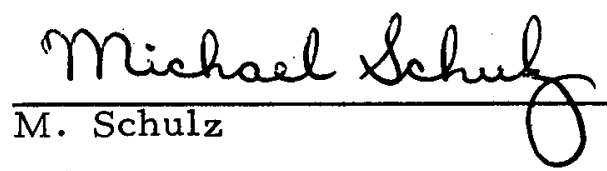

J.M. Cormunall

J. M. Cornwall
YB Blake

J. B. Blake, Head

Space Particles and

Fields Department 


\section{THIS PAGE \\ WAS INTENTIONALLY \\ LEFT BLANK}


ABSTRACT

This is the final report of an initial assessment of magnetospheric effects of the construction and operation of a satellite power system. This assessment effort is based on application of present scientific knowledge rather than on original scientific research. As such, it appears that mass and energy injections of the system are sufficient to modify the magnetosphere substantial$1 y$, to the extent of possibly requiring mitigation measures for space systems but not to the extent of causing major redirection of efforts and concepts. The scale of the SPS is so unprecedentedly large, however, that the se impressions require verification (or rejection) by in-depth assessment based on scientific treatment of the principal issues. Indeed, it is perhaps appropriate to state that present ignorance far exceeds present knowledge in regard to SPS magnetospheric effects, even though we only seek to define the approximate limits of magnetospheric modifications here. 


\section{THIS PAGE \\ WAS INTENTIONALLY \\ LEFT BLANK}


CONTENTS

ABSTRACT .......................... v

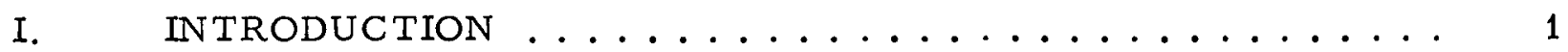

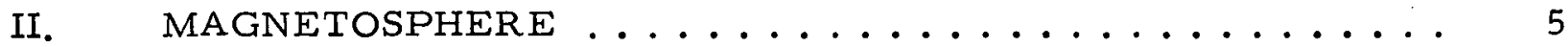

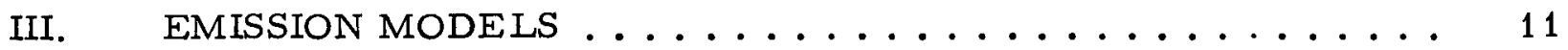

IV. ARGON ION ENGINE EXHAUST (COTV) IN

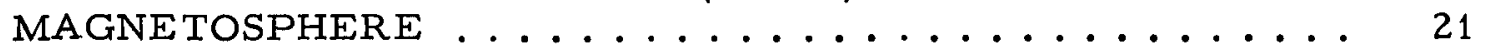

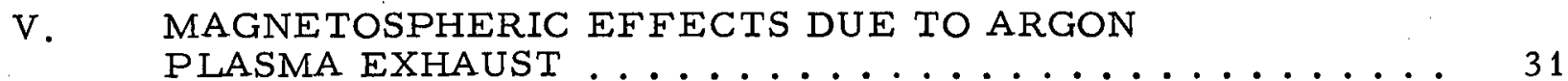

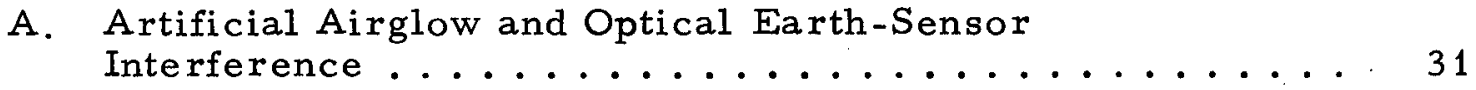

B. Ionospheric and Plasmaspheric Heating .......... 36

C. Ring Current Modifications ................ 40

D. Radiation Belt Modification ............... 46

E. Plasma Instabilities and Effects on Space

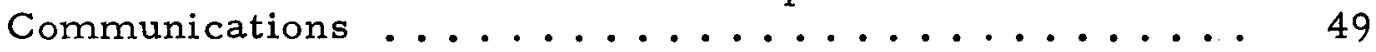

VI. EFFECTS OF NEUTRAL EXHAUST (POTV) .......... 55

VII. SUMMARY OF MAGNETOSPHERIC EFFECTS ......... 59

VIII. RECOMMENDATIONS FOR FINAL ASSESSMENT . . . . . . . 63

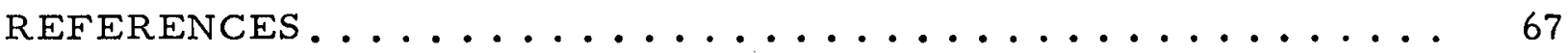




\section{THIS PAGE \\ WAS INTENTIONALLY \\ LEFT BLANK}

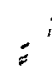

$i$ 


\section{FIGURES}

1a. Structures in Magnetosphere Taken in Cross Section on Noon-Midnight Meridional Plane ..............

1b. More Simplified View of Magnetosphere Taken on

Same Cross Sectional Plane as in Figure 1a .........

2. Schematic Representation of Cargo Orbit Transfer Vehicle (COTV) Argon-Ion Emission Scenario

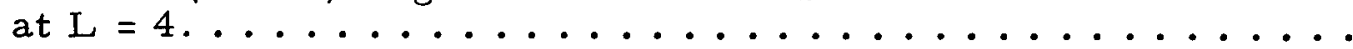

3. Spiral Orbit Trajectory of COTV from LEO

(Innermost Circle) to GEO (Outermost Circle),

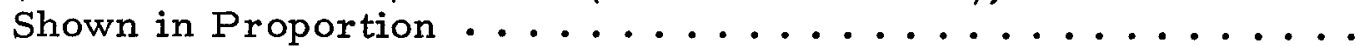

4. Argon Propellant Mass Necessary for Transport of

Different Payload Masses between $350 \mathrm{~km}$ Altitude

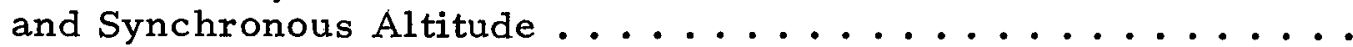

5. Time Fraction of LEO-GEO Transfer Orbit at

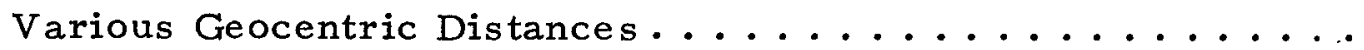

5a. Number of $\mathrm{Ar}^{+}$Ions Released at Various Geocentric

Distances for Payload Mass of $10 \mathrm{~kg}$, Compared

to Number of Ambient Electrons in a Dipole Field

Shell Two Argon Gyroradii Thick. ................

6. Critical Pitch Angle (Charge-Exchange Loss Cone

Angle) Shown as Function of Radial Distance from

LEO at Various L-Values...................

7. Schematic Representation of Beam-Magnetosphere and

Beam-Ionosphere Interactions Involving Propagation

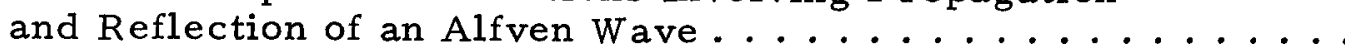

8. Alfven Speed Shown as Function of Radial Distance from

Earth's Surface for Geomagnetic Field Lines of Various

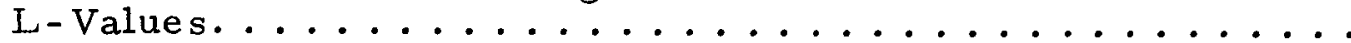

9. Comparison of Charge Exchange and Coulomb Lifetimes of $\mathrm{Ar}$ Ions at Various Geocentric Distances in

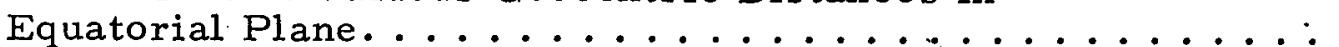


FIGURES (Continued)

10. Comparison of Compositional Structure of Plasmasphere Under Natural Thermal Conditions and Under Heated

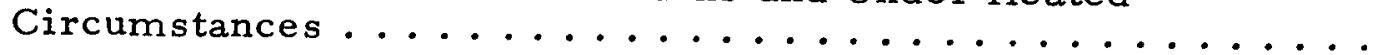

11. Lowest Order Estimates for Normalized Growth Rate as Function of Normalized Wave Frequency, for Various Relative Concentrations $\left(\mathrm{N}_{\mathrm{Ar}} / \mathrm{N}_{\mathrm{H}}\right)$ of Argon

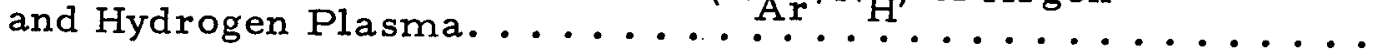

12. Product $(\sigma v)$ of Charge-Exchange Cross Section, $\sigma$, (between Indicated Ions and Neutral Hydrogen Atoms) and Ion Speed,v, Shown as Functions of Ion Energy ......... 
TABLES

I. Ion Engine Characteristics .................. 12

II. Artifical and Natural $\mathrm{N}_{2}^{+}(2500-5500 \mathrm{~A})$

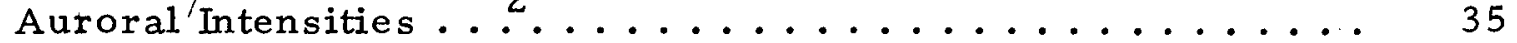

III. Instabilities Relevant to SPS Plasma Injection . . . . . . . . 50

IV. Satellite Power System Magnetospheric Effects . . . . . . . . 61 


\section{INTRODUCTION}

The space segment of the satellite power system (SPS) is projected to operate at geosynchronous orbit (GEO) at an altitude of $\sim 36,000 \mathrm{~km}\left[\sim 6.6\right.$ earth radii $\left(R_{E}\right)$ from the center of the earth] in the equatorial plane. During the spacecraft construction phase, transportation and assembly activities will take place between low earth orbit (LEO) at $\sim 400 \mathrm{~km}$ altitude and GEO. Thus, the operational activities of the SPS and a major segment of the constructional activities take place in the magnetosphere, which is a region of near-earth space where the ionized medium is controlled by magnetic and electric fields. These activities in space represent a loading upon the tenuous but spatially vast magnetospheric environment. The magnitude of this loading, in the form of injections of matter and energy, can be qualitatively accentuated by comparison of the mass of the SPS spacecraft $\left[\sim(3-5) \times 10^{7} \mathrm{~kg}\right]$ at GEO with the largest spacecraft operated to date [Skylab, $7 \times 10^{4} \mathrm{~kg}$ ] at LEO. While Skylab did not seem to have any appreciable effects upon the magnetosphere, the key question, however, is whether the scaling-up of spacecraft mass by three orders of magnitude and the scaling-up of orbit altitude by two orders of magnitude would have appreciable magnetospheric effects. Further, if magnetospheric modifications were expected, what mitigation strategies would be required in system design?

The primary goal of the assessment effort is to evolve an answer to the above key questions. This report represents our initial assessment of the magnetospheric ef fects of SPS based upon application of present knowledge and is written with a view toward recommendations for future studies and observations needed to verify the assessments. As such, we have focused our efforts to assess the entire range of magnetospheric effects which may conceivably be of importance, rather than to do original research-indepth upon particular issues. In-depth assessment of the more important issues will be considered as follow-on studies af ter their priorities have been determined by simple but physically appropriate assessments of the present study.

As is implicitly noted in the SPS-Skylab comparison above, a number of uncertain factors necessarily underlie the assessment of magnetospheric effects; the primary reason being that the mass scale and the altitude range of construction and operation activities of the SPS are unprecedented. In this study, theoretical knowledge is applied to bridge this gap in scaling; however, it must be noted at the outset that a second-stage effort towards the goal of final assessment of magnetospheric effects must necessarily 
involve follow-on in-depth assessment studies and experimental verification of the theoretical results considered here. Indeed, we may view our efforts here as exploration and selection of issues so that a coherent program of priorities can be constructed for experimental verification of SPS magnetospheric effects assessment.

Obviously, our study would run the risk of being too shallow if we attempt to cover the entire multitude of identified magnetospheric issues. We consequently focus our efforts primarily upon the issues connected with the injections of free energy and of mass into the magnetosphere (including the evolution of the injected free energy) because we deem these issues to be of more immediate concern to human activities in space and on the ground. Included in our consideration are, among others, the magnetospheric injections of solar electric propulsion vehicles, which use ionized argon gas as propellant, and of chemical propulsion vehicles, which would release neutral hydrogen and oxygen in the magnetosphere. Effects induced by these magnetospheric modifications include radiation belt dosage modification, optical earth-sensor interference, possible communication interference and possible modification of the atmospheric electric circuit which may impact powerline and pipeline operations. The severity of these impacts is much more difficult to determine with a high degree of certainty without in-depth scaling up to SPS proportions. Indeed, it can be said that we have uncovered a higher degree of present ignorance than present knowledge insofar as the scaling of impacts up to the SPS level is concerned. Of those cases that we were able to consider within limits of present knowledge, it is probably fair to say that the impacts are of sufficient concern to induce consideration of design and mitigation strategies, but are probably not so severe as to put a stop to the project. We remind the reader, however, that these are present impressions on some specific issues and are subject to future rejection when (and if) new results are obtained.

The report is organized into 8 Sections. Section II introduces the reader to relevant features and physical processes of the magnetosphere. Readers already familiar with the magnetosphere can skip this section without loss of continuity. Section III is an attempt to quantify the injection sources into emission models. Section IV discusses the basic physics of the main interactions involved in argon ion injections into the magnetosphere. The separate magnetospheric effects upon human activities, caused by the character and quantity of argon plasma injections treated in Sections III and IV, are discussed in five Subsections of Section V. In Section VI, we introduce the reader to the 
significance of neutral chemical exhaust of the personnel orbit transfer vehicles (POTV) in the magnetosphere. Assessment of neutral exhaust effects were not originally called for in the FY79 contract but their significance was realized in the course of this assessment effort. A brief summary and assessment of magnetospheric effects is given in Section VII. Recommendations for the completion of final assessments are considered in Section VIII. Sections VII and VIII can be perused as an integral unit of magnetospheric effects assessment (skipping Sections IV, V and VI) by readers who are interested in the salient, rather than detailed physical, features of this report. 
THIS PAGE

\section{WAS INTENTIONALLY \\ LEFT BLANK}




\section{MAGNETOSPHERE}

In this section, we shall give a brief description of the relevant features of the magnetospheric environment. In the process, the reader is introduced to the technical terms used in the text. Readers already familiar with the magnetosphere can skip this section without loss of continuity.

The solar wind, a supersonic plasma flow (typical density $\sim 8 \mathrm{~cm}^{-3}$ and speed $\sim 300$ $\mathrm{km} / \mathrm{sec}$ at earth orbit) away from the sun, is deflected by the earth's magnetic field at a stand-off distance of $\sim 10 \mathrm{R}_{\mathrm{E}}$ in the sun-ward direction. Equivalently, the earth's magnetic field is said to be confined to a cavity called the magnetosphere by the solar wind (Figure 1a). On the night side, the magnetospheric cavity extends some hundreds of earth radii downstream where present evidence seems to indicate a merging of the earth's magnetic field lines with that of the solar wind magnetic field. This geomagnetic tail region is bisected near the equatorial plane by the neutral sheet where the magnetic field lines originating from the north and south polar caps cancel each other creating a dawn-dusk current sheet (Figure 1a). The electric field (dawn-dusk) associated with this current sheet is called the convection electric field because the hot plasma in the vicinity of the neutral sheet (plasma sheet) is convected sun-ward by the $\vec{E} \times \vec{B}$ force. The earthward end of the plasma sheet is connected by magnetic field line to the auroral zones. In the course of a magnetic storm, solar wind plasma enters into the plasma sheet and is driven earthward on the night side by the increased convection electric field, forming a ring current of hot plasma in the vicinity and earthward of GEO, where SPS is stationed (Figure 1b): Precipitation of the hot ring current plasma along dipole-like magnetic field lines into the auroral zone causes atmospheric airglow at $\sim 100 \mathrm{~km}$ altitude known as the aurorae. Note that the operating location of SPS on the nightside is in the key region where the auroral process takes place in response to solar wind disturbances (Figure 1b).

As one moves earthward of GEO, the geomagnetic field configuration becomes increasingly less distorted by the solar wind and more dipole-like. At a radial equatorial distance of about $4 \mathrm{R}_{\mathrm{E}}$, one encounters a distinct plasma boundary, the plasmapause, which marks the surface of the plasmasphere. The plasmasphere is a dipole-like volume inside of which the thermal plasma density is several orders of magnitude higher than magnetospheric regions outside. Indeed, there is increasing evidence that the plasmasphere is just a continuation of the topside ionosphere which is trapped by the geometry 
of magnetic and electric fields. The processes of particle trapping and diffusion in the dipole-like magnetic field of this region are conducive to long-lived accumulation of injected matter and energy. We shall consider some of these processes in this report.

Aside from the cold plasma of ionospheric origin and hot plasma of solar wind origin, solar and galactic cosmic ray processes and magnetospheric energization give rise to a trapped component of energetic particles (electrons, protons and alpha particles) which undergo trapped adiabatic motion in the plasmasphere. These particles are primarily concentrated in two torus-like shells known as the Van Allen radiation belts (Figure 1a). Since the natural radiation dosage in these belts are hazardous to humans and to space-borne equipment, radiation belt dosages represent design limits for space systems which operate in this region. For example, for manned lunar missions the thrusting schedule has to be carefully designed to avoid excessive dosage in traversing the radiation belts. Thus, any magnetospheric modification by SPS injectants must be considered in the light of their possible effects upon the radiation belt dosage. Fortunately, because of their practical importance and because of large scale modification experiments in the past, e.g., the Starfish nuclear event, the physics of radiation belt processes is relatively better understood (Schulz and Lanzerotti, 1973); and we are able to make relatively more definitive assessments in this area.

As is described in the morphological features above, magnetospheric particles are not entirely isolated from interactions with their neighbors in the ionospheric and atmospheric regions below. Indeed, these interactions control the lifetime and energy evolution of magnetospheric particles. For this reason, it would be convenient to introduce some basic ideas concerning particle motion in the magnetosphere.

The magnetosphere, including the denser plasmasphere, is sufficiently tenuous that energetic particles move under the influences of the dipole-like magnetic field and electric fields without consideration of collisions. A collision, most probably a chargeexchange interaction with the more abundant neutral species existing in the magnetosphere and in the upper layers of the atmosphere, usually means loss of the charged energetic particle from the magnetosphere (precipitation). Thus, the lifetime $\tau$ of a charged particle is characterized by the inverse rate of charge-exchange interaction,

$$
\tau=1 / \mathrm{N}_{\mathrm{n}} \sigma \mathrm{v}
$$




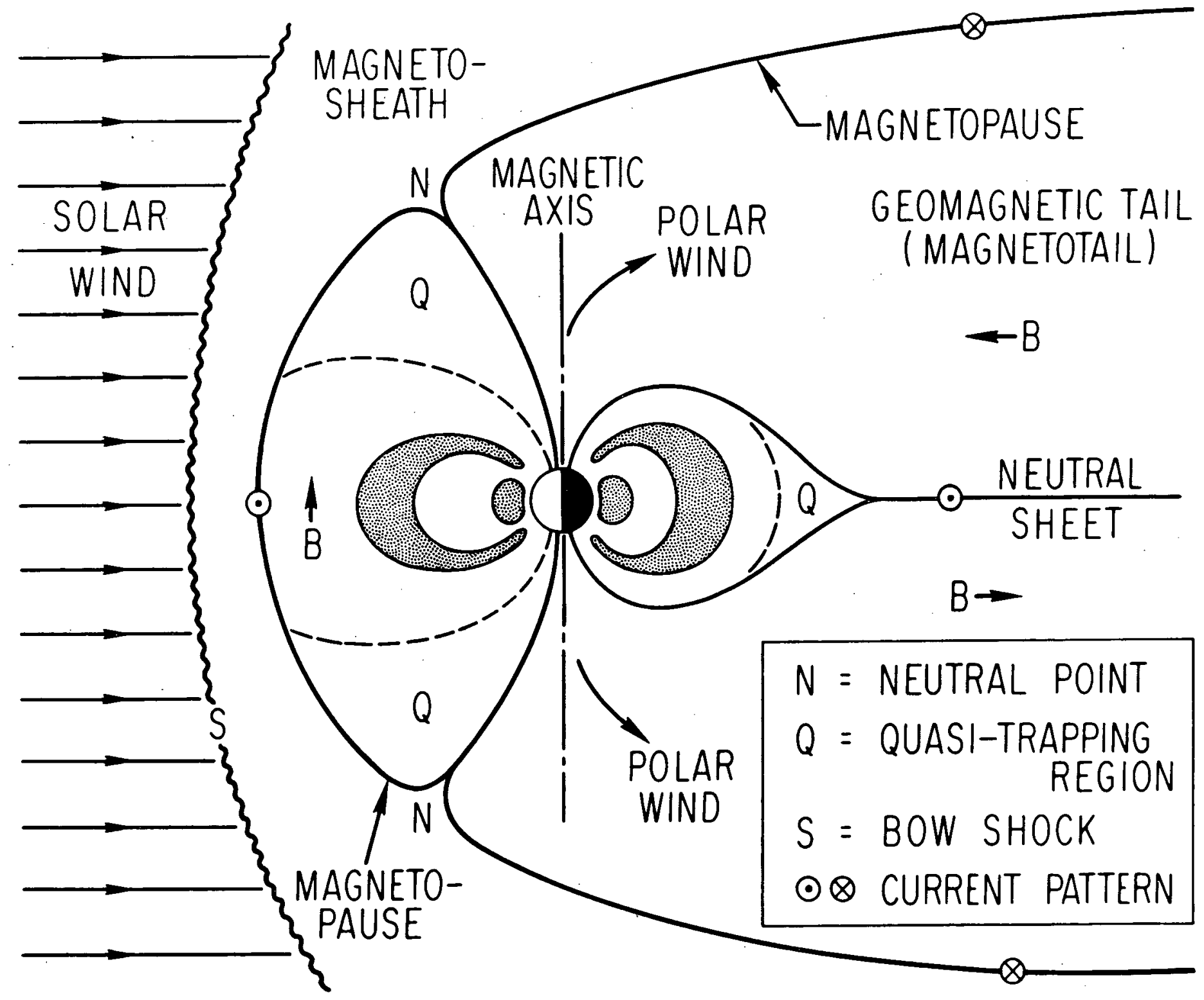

Figure 1a. Structures in Magnetosphere Taken in Cross Section on Noon-Midnight Meridional Plane. Stipled areas show the two quasi-toroidal belts of trapped radiation. 


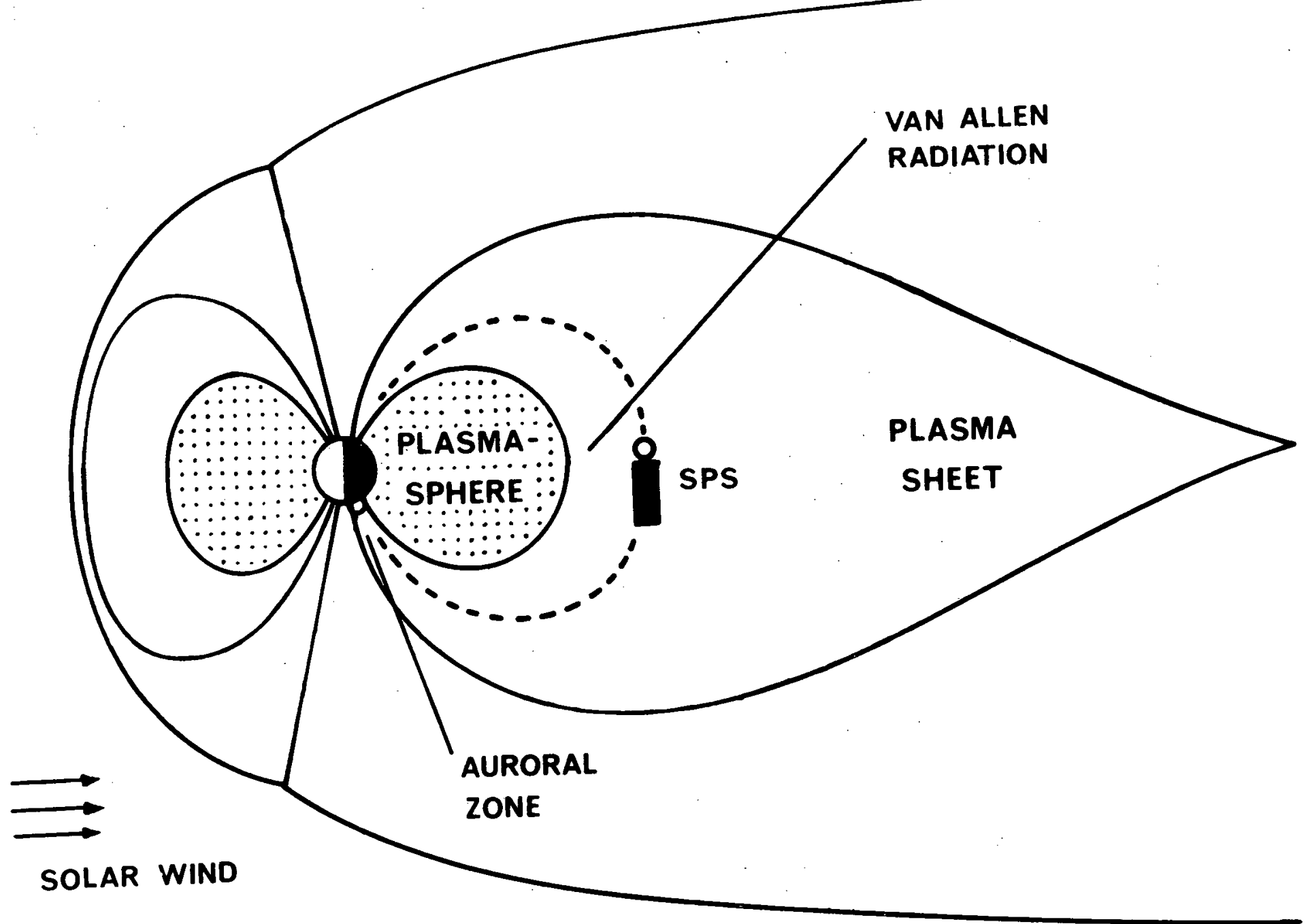

Figure 1b. More simplified View of Magnetosphere Taken on Same Cross Sectional Plane as in Figure 1a. Location of SPS in relation to features relevant to magnetospheric effects is shown. 
where $N_{n}$ is the neutral density involved (requiring various sorts of averaging over the volume of interaction), $\sigma$ is the charge exchange cross-section and $v$ is the characteristic charged particle speed involved. At the lower regions of the magnetosphere (footof-field-line), $N_{n}$ rapidly e-folds over a typical neutral scale height $H \sim 20 \mathrm{~km}$; hence, the altitude $z_{c e}$, determined by the functional relation

$$
\sigma \mathrm{N}_{\mathrm{n}}\left(\mathrm{z}_{\mathrm{ce}}\right) \mathrm{H}\left(\mathrm{z}_{\mathrm{ce}}\right)=1 \text {, }
$$

marks the vicinity where energetic particle interactions with the atmosphere (such as airglow emissions) occur.

Electric fields $\vec{E}$ in the magnetosphere (with exception in the auroral region) are primarily perpendicular to the magnetic field $\vec{B}$. Under the influence of these two fields, a charged particle drif ts with velocity $\vec{v}_{D}$ perpendicular to both $\vec{E}$ and $\vec{B}$

$$
\overrightarrow{\mathrm{v}}_{\mathrm{D}}=\mathrm{c} \overrightarrow{\mathrm{E}} \times \overrightarrow{\mathrm{B}} /|\mathrm{B}|^{2}
$$

In addition, the charged particle executes its usual gyration motion in the magnetic field with a gyration frequency $\Omega=\mathrm{eB} / \mathrm{mc}$ and a gyroradius (Larmor radius) $\rho=\mathrm{v}_{\perp} / \Omega$. Since the geomagnetic field is dipole-like and particle velocity generally has parallel $\left(v_{\|}\right)$and perpendicular components $\left(v_{\perp}\right)$, charged particles in the magnetosphere execute a gyrating-bouncing motion in much the same way as ions in a magnetic bottle. The location on a magnetic field line (customarily labelled by its equatorial geocentric distance $R$ in units of $R_{E}, L \equiv R / R_{E}$ ) where the particle bounces back is known as the mirror-point. If the mirror-point of a charged particle happens to be below the altitude $z_{\text {ce }}$, the particle charge-exchanges and never returns to its former trapped motion in the magnetic field. The mirror-point of a particle depends on its initial velocity $\vec{v}$ with respect to the magnetic field; in particular, the mirror-point is determined by the initial pitch-angle $\alpha_{0} \equiv \cos ^{-1}\left(v_{\|} /|v|\right)$ where $v_{\|}$is the component of $\vec{v}$ parallel to the magnetic field. In other words, if an ion is injected at a geocentric distance $r_{0}$ on a given dipole field line $\mathrm{L} \equiv \mathrm{R} / \mathrm{R}_{\mathrm{E}}$, then we can determine a critical initial pitch-angle $\alpha_{\text {crit }}$ such that all particles with initial pitch-angles $\alpha \leq \alpha_{\text {crit }}$ will have mirror-points below a given geocentric distance $r_{\ell}$ on the same field line 


$$
\alpha_{\text {crit }}=\sin ^{-1}\left\{\left[\frac{4 R-3 r_{0}}{4 R-3 r_{\ell}}\right]^{1 / 4}\left[\frac{r_{\ell}}{r_{0}}\right]^{3 / 2}\right\}
$$

If $r_{\ell}$ is identified with the precipitation altitude $z_{c e}$, then $\alpha_{\text {crit }}$ is called the loss cone angle because all particles with pitch angle less than $\alpha_{\text {crit }}$ are "lost". According to (4), if an ion is injected at LEO $\left(r_{0} \approx r_{l}\right)$ with $v_{\|} \neq 0$, it will almost certainly be lost, because $\alpha_{\text {crit }} \approx 90^{\circ}$ at LEO. Conversely, at GEO $\left(r_{0} \gg r_{\ell}\right)$ the loss-cone tends towards $0^{\circ}$; hence, only those ions injected with $\vec{v}$ almost entirely parallel to the magnetic field are lost. Such considerations are very important in assessing a number of effects such as airglow intensity and plasma instabilities generated by SPS spacecraft exhaust emissions.

The lower boundary of the magnetosphere is, strictly speaking, the spherical ionospheric layer known as the E-layer in which particle motion is no longer controlled by the magnetic field. However, for purposes of SPS assessment, ionospheric effects are dealt with elsewhere, therefore, we shall regard LEO as the lower boundary altitude of the magnetospheric effects, although because of magnetospheric-atmospheric interactions such a common lower boundary does not exist for all magnetospheric processes. 


\section{EMISSION MODELS}

In the present section, we attempt to describe the characteristics of the SPS injectants in the magnetosphere. The description is limited to the determinable quantitative parameters from the reference system report of the SPS concept development program (U.S. DOE, 1979) and the SPS Baseline Review (U.S. NASA, 1978). The evolution of each injectant in the magnetosphere is not considered in this section, because the evolution is properly the magnetospheric effect itself. Even for this limited task at hand, we have found that the information available is not sufficient for us to completely evaluate the impacts on specific physical processes. This is particularly true in regard to thrusting schedules of the spacecraft engines. We have attempted to supplement the basic information with some consideration of rocket dynamics.

The primary sources of matter and energy injected into the magnetosphere are ionized argon exhaust from the solar-powered cargo orbit transfer vehicles (COTV) that ferry the payload between LEO and GEO, and neutral chemical exhaust in the form of $\mathrm{H}_{2} \mathrm{O}$ (or $\mathrm{H}$ and $\mathrm{O}$ atoms after photodissociation) from the personnel orbit transfer vehicles (POTV). In addition, the COTV may also use subsidiary chemical engines and the SPS spacecraft in its operational form may use argon ion ( $\mathrm{Ar}^{+}$) engines for stationkeeping activities. Because of this simple break-down into two key elements of magnetospheric injection and because the present assessment effort is not expected to represent a complete scaling-up of physical phenomena to SPS dimensions, we shall consider the emissions in terms of output from a single SPS mission, rather than a sequence of SPS missions.

The current technology of ion engines is still evolving (Kauffman, 1974; Byers and Rawlin, 1976); therefore, the parameters of argon-ion engine operations in space must largely be regarded as uncertain at present, although it is by now fairly firm that the most economical and environmentally safe propellant is argon. According to the reference system report of the satellite power system concept development and evaluation program (U.S. DOE, 1979) argon-ion engines of specific impulse 13000 sec. are projected for the COTV. Other considerations (Byers and Rawlin, 1976), with perhaps less stringent requirements on projected advances on the technology of ion engines, assumed 5000 sec. specific impulse as standard for comparison. Some projected characteristics of these two options of ion engine operation are listed in Table 1 , in which Option I (the official concept option) will be used as reference in this report. 
Table I. Ion Engine Characteristics

Option 1

13000

3.5

130

$2.5 \times 10^{-2}$

$\sim 1000^{*}$

$\sim 1.5 \times 10^{10}$

100

$\sim 10^{\circ}$

$\sim 300$
Option 2

5000

0.5

50

$2.5 \times 10^{-2}$

$\sim 1000^{*}$

$\sim 4 \times 10^{10}$

100

$\sim 10^{\circ}$

$\sim 800$

*Kauffman (1974) 
From Table I, it is seen that the ion beam exhaust is a very dense but fairly cool plasma whose streaming kinetic energy (3.5 keV) far exceeds the thermal energy. In order to propel the COTV to geosynchronous orbit, the argon plasma beam will be directed primarily perpendicular to the geomagnetic field at the equatorial plane in the azimuthal direction (Figure 2). The COTV transfer orbit will be a spiral in the equatorial plane (Figure 3). Plasma beams propagating perpendicular to the geomagnetic field entail very interesting dynamical interactions with the magnetosphere-ionosphere system; in this case, this interaction takes place not at a single location but at all equatorial altitudes from LEO to GEO (Figures 2 and 3). Although, for purposes of considering large-scale magnetospheric modification, the argon plasma beam can be regarded as being perpendicular to the magnetic field at the equatorial plane, it is technically not entirely correct because the magnetic axis is off-set from the earth's spin axis and because proposed SPS launch site at Florida would necessitate a $28.5^{\circ}$ orbit inclination change during transfer orbit to GEO. The exact orientation of the argon plasma beam with respect to the local magnetic field depends entirely on the above factors and on the details of the planned thrusting schedule, which is not available. Considerations of particle motion in the previous section indicate that this exact orientation is not of great significance for global scale environmental assessment except at the vicinity of LEO where direct precipitation of a dense beam of $3.5 \mathrm{keV} \mathrm{Ar}^{+}$would cause airglow (artificial aurora) emissions and atmospheric heating far exceeding that of the natural aurorae. These effects will be discussed more fully in Subsection V.A.

For consideration of argon plasma emission parameters, Figure 4 (taken from Chiu et al., 1979a) shows the relationship between payload mass and argon propellant mass needed to transport the payload from LEO $(\sim 400 \mathrm{~km}$ altitude) to synchronous altitude with an accompanying orbital plane change of $28.5^{\circ}$. Obviously, the amount of propellant required for a given payload depends on the ion-beam streaming speed. For an SPS payload of $\sim 4 \times 10^{7} \mathrm{~kg}$, it will be necessary to expend $\sim 10^{6} \mathrm{~kg}$ of argon propellants for option 1 in Table 1 . This is $\sim 2 \times 10^{31} \mathrm{Ar}^{+}$ions, roughly comparable to the total content of the natural plasmasphere and ionosphere above $500 \mathrm{~km}$. The exhaust deposition rate in terms of the time fraction of LEO-GEO transfer orbit, which is nominally $\sim 130$ days, is shown as a function of geocentric radius $R$ on Figure 5 (Chiu et al., 1979a). Thus, 80 percent of the total propellant content is released in the plasmasphere, $R \leq 4 R_{E}$ (Figure 3). The number of $\mathrm{Ar}^{+}$released at a given geocentric distance for a payload mass of $4 \times 10^{7} \mathrm{~kg}$ is shown on Figure 5a (Chiu et al., 1979c); for 
comparison, the number of ambient electrons lying within a flux shell of thickness equal to twice the argon gyroradius at a given distance $R$ is also shown. The energy content released into a given shell dominates the ambient energy content, however, since the streaming $\mathrm{Ar}^{+}$kinetic energy is $3.5 \mathrm{keV}$ and the ambient thermal energy is $\sim 1 \mathrm{eV}$.

Argon ion engine exhaust in the magnetosphere is thus unique in that it represents both matter and energy injections. The evolution of these two forms of injections follows very different paths, as we shall show.

Neutral chemical exhaust is initially emitted in the magnetosphere as $\mathrm{H}_{2} \mathrm{O}$ molecules in the exhaust of $\mathrm{LO}_{2} / \mathrm{LH}_{2}$ rocket engines of the POTV. In the magnetosphere, the $\mathrm{H}_{2} \mathrm{O}$ molecules are expected to be photodissociated into $\mathrm{H}$ and $\mathrm{O}$ atoms. Since chemical rocket combustion temperatures are $\sim 2000^{\circ} \mathrm{K}$, slightly less but comparable to the ambient magnetospheric temperature, chemical rocket exhaust does not represent injection of extra free energy, just injection of matter.

According to the SPS baseline concept review (U.S. NASA, 1978) the POTV engine assembly consists of two stages with 4 engines in the first stage and 2 engines in the second stage. Each engine has a mass flow rate $F \simeq 104 \mathrm{~kg} / \mathrm{sec}$. Because no thrusting schedule is given, we cannot determine the scheduled flow rate. Taking an averaging approach, one may assume as a working model average total POTV exhaust rate $\mathrm{F} \simeq 310$ $\mathrm{kg} / \mathrm{sec}$ of $\mathrm{H}_{2} \mathrm{O}$ over an average exhaust cross-section of $\sim 9 \mathrm{~m}^{2}$ at exit (average of 3 nozzles of $2 \mathrm{~m}$ diameter each). The exhaust speed is $\mathrm{u} \sim 4 \mathrm{~km} / \mathrm{sec}$. Once the exhaust leaves the nozzle it expands primarily isotropically away from the exhaust beam axis. Thermodynamically, this expansion is controlled by the temperature of the exhaust so that relatively more molecules have zero transverse speed and the abundance of molecules with higher transverse speeds falling off in a Gausian fashion according to a thermal distribution. The proper treatment of the density distribution in such a thermally expanding jet in collisionless medium is by solution of the Boltzmann equation. Such an exact solution of the free expansion of a neutral jet is given in Subsection V.A. Since the assessment of the effects of neutral POTV exhaust is not originally called for in the FY79 contract activity, we have not applied such a model of the neutral jet to the limited assessment of POTV neutral exhaust effects given in this final report. Consequently, the mode of free jet expansion is given in Subsection V.A. Instead, an averaging approach can be used to estimate the neutral jet characteristics here. Assuming the exhaust to expand away from the beam axis at an average speed $w \sim u / 10 \simeq 0.4$ $\mathrm{km} / \mathrm{sec}$, the neutral exhaust cloud would occupy an approximately conical volume $\mathrm{V}=$ 


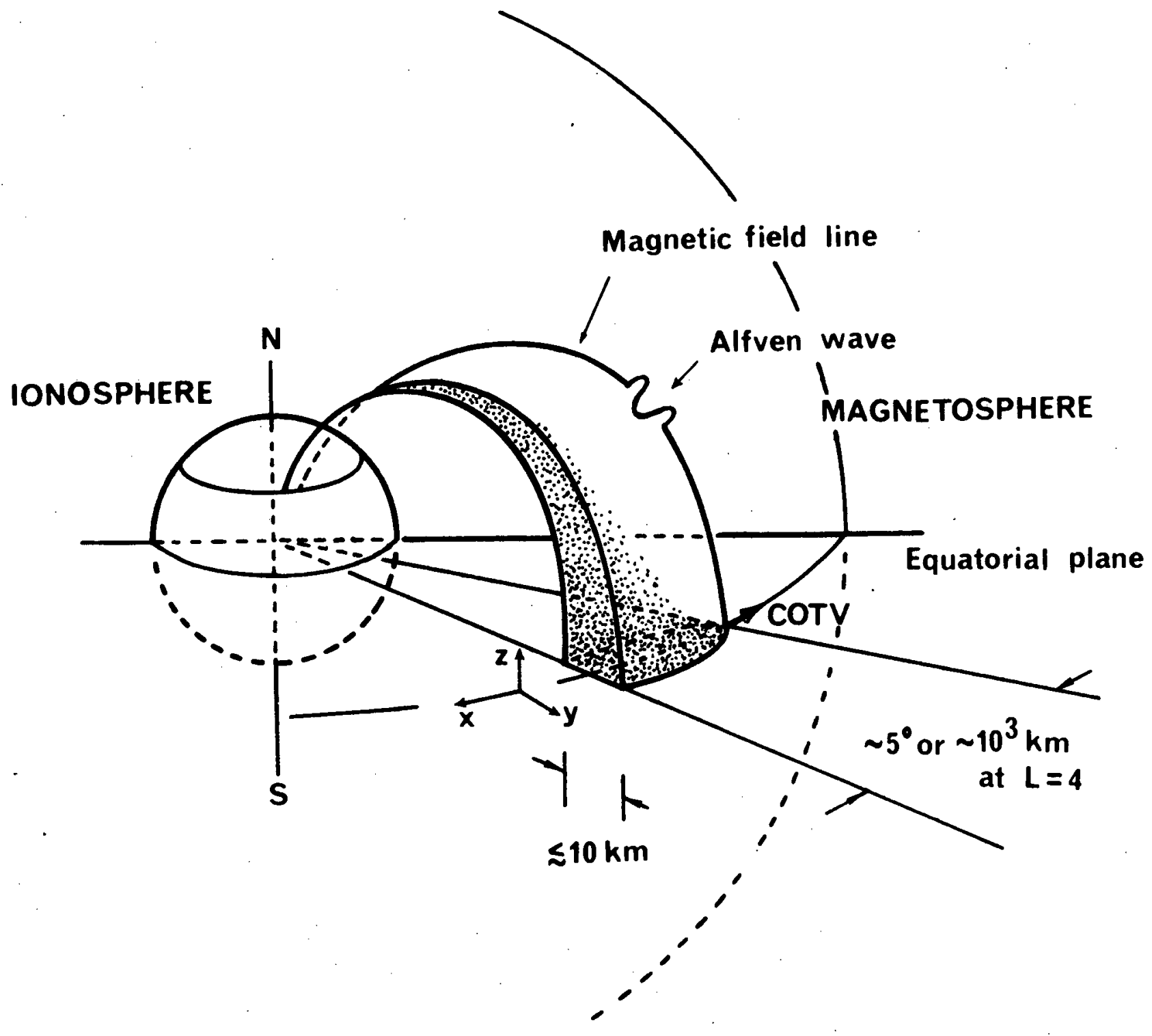

Figure 2. Schematic Representation of Cargo Orbit Transfer Vehicle (COTV) Argon-Ion Emission Scenario at $\mathrm{L}=4$. Coordinate system used in this paper is also defined. Ion beam trailing the COTV is roughly $1000 \mathrm{~km}$ long and $\sim 10 \mathrm{~km}$ in width. 


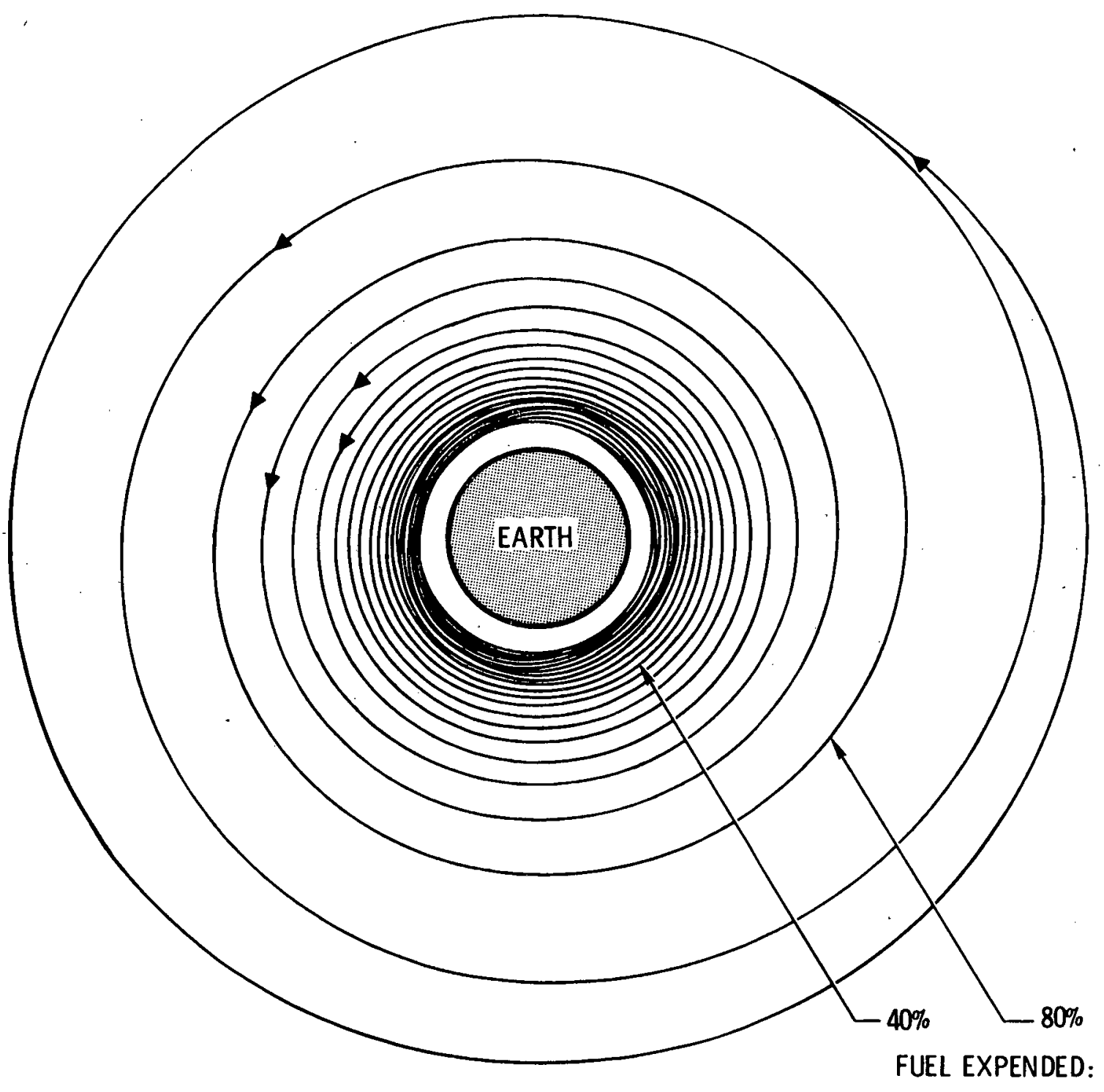

Figure 3. Spiral Orbit Trajectory of COTV from LEO (Innermost Circle) to GEO (Outermost Circle), Shown in Proportion. 


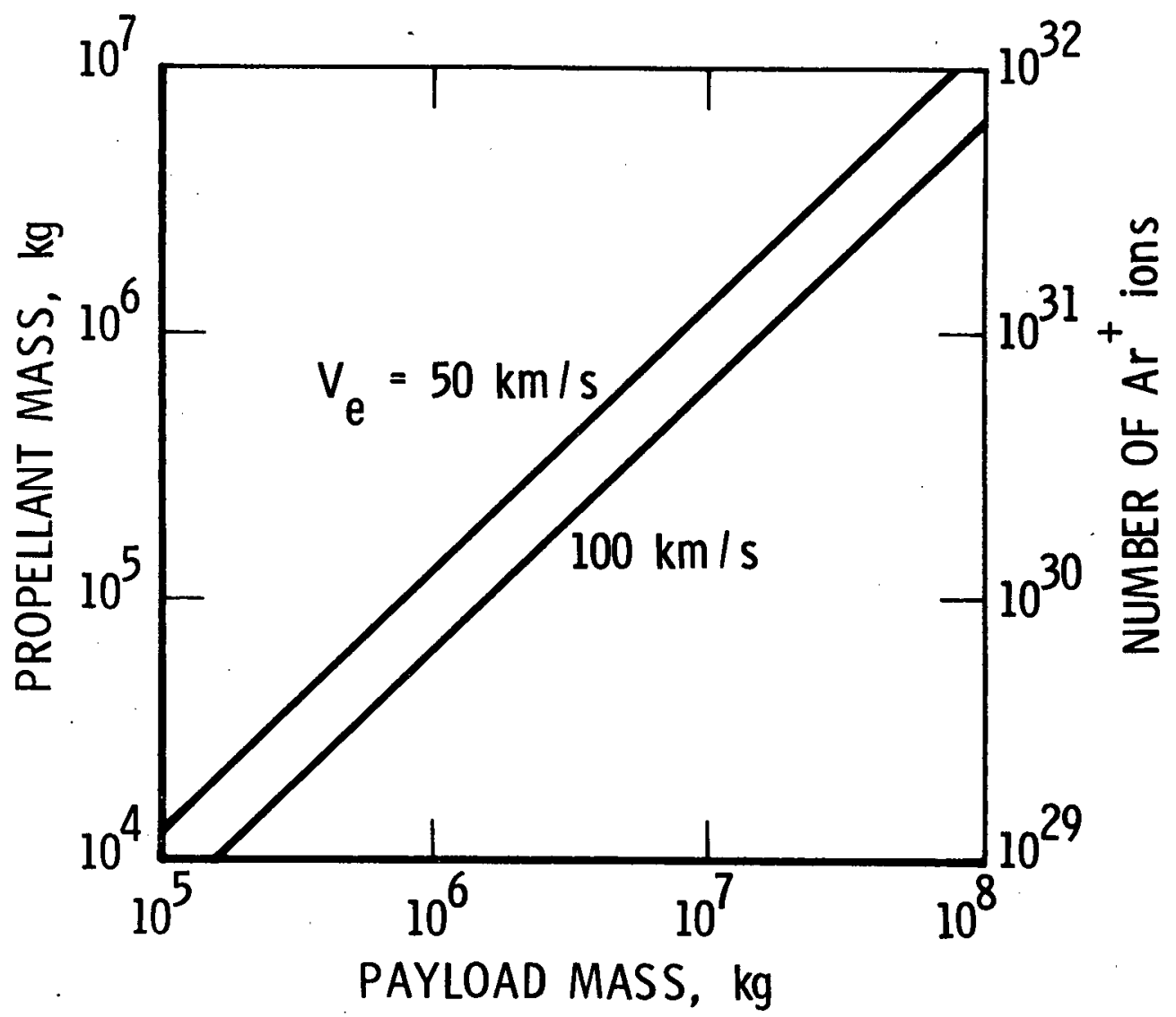

Figure 4. Argon Propellant Mass Necessary for Transport of Different Payload Masses between $350 \mathrm{~km}$ Altitude and Synchronous Altitude. Orbital inclination is assumed to change from $0^{\circ}$ to $28.5^{\circ}$ during transport. Projected exhaust speed of $130 \mathrm{~km} / \mathrm{sec}$, corresponding to $3.5 \mathrm{keV}$ kinetic energy, is not shown but should fall just below the curve for $100 \mathrm{~km} / \mathrm{sec}$. 


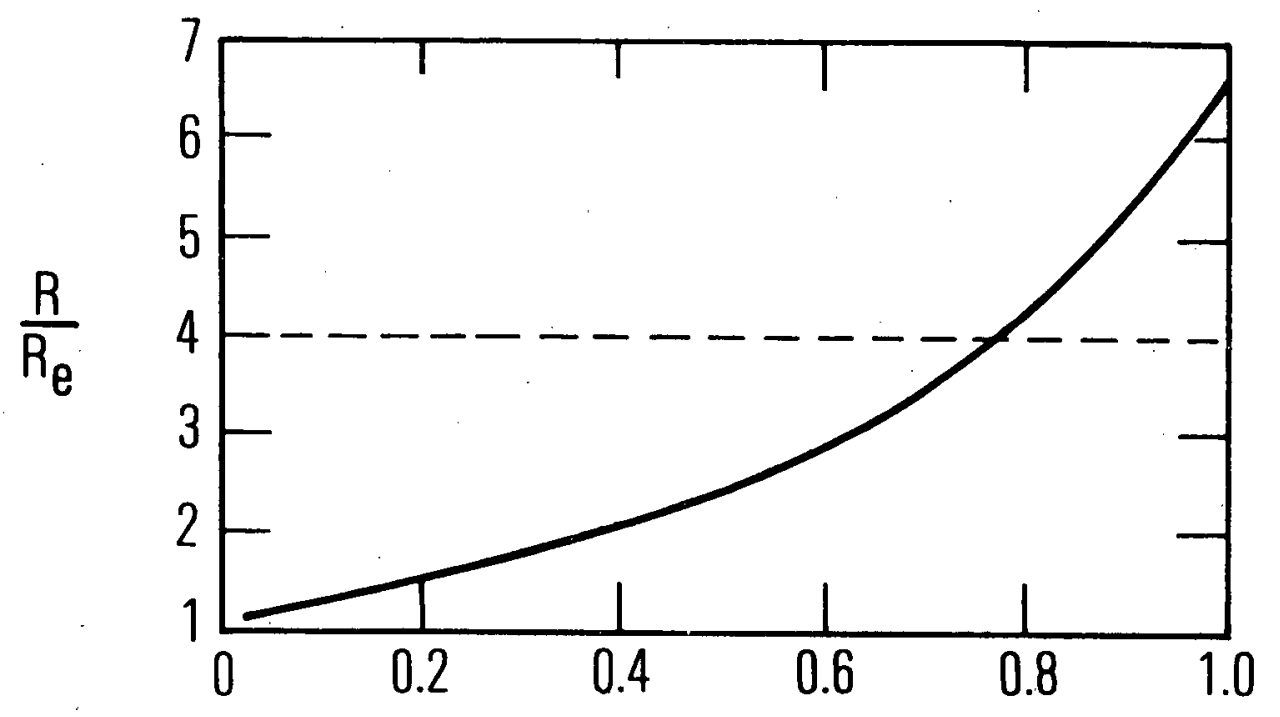

Figure 5. Time Fraction of LEO-GEO Transfer Orbit at Various Geocentric Distances 


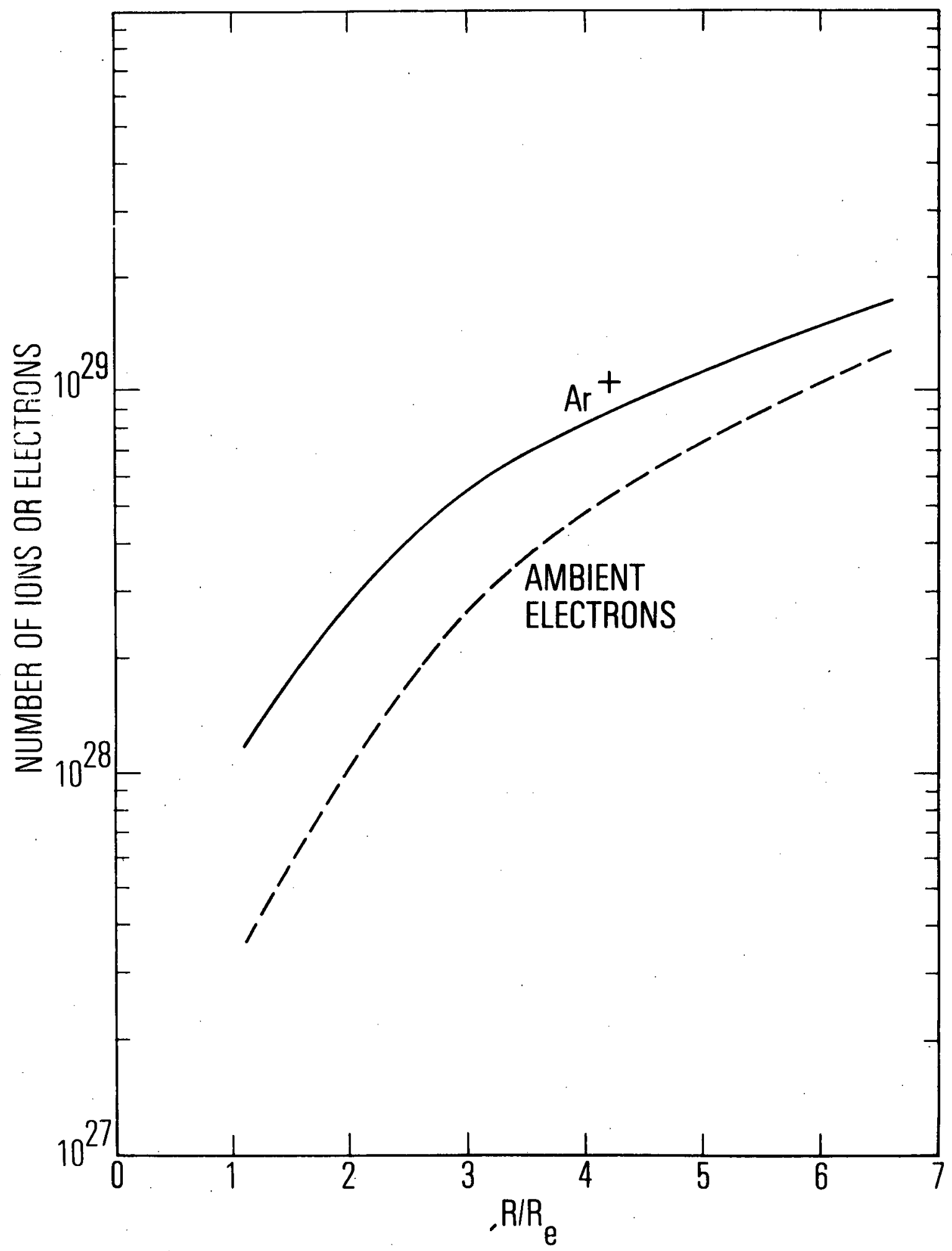

Figure 5a. Number of $\mathrm{Ar}^{+}$Ions Released at Various Geocentric Distances for Payload Mass of $10 \mathrm{~kg}$, Compared to Number of Ambient Electrons in a Dipole Field Shell Two Argon Gyroradii Thick 
$\pi u^{2} \mathrm{w}^{3} / 3$ at any time $\mathrm{t}>0$. The density distribution $\mathrm{N}$ inside this volume depends on the details of the expansion as described above. For our purposes here an average estimate is $N \sim F t / V$. This would imply an average density of $\sim 10^{13} /\left(4 t^{2}\right)$ atoms $/ \mathrm{cm}^{3}$, where $t$ is measured in seconds. As we shall see, such a dense neutral cloud in the magnetosphere would significantly influence the lifetimes of charged particles in the magnetosphere.

We have attempted to collect and summarize the available information on emission parameters of argon ion engines and of $\mathrm{LO}_{2} / \mathrm{LH}_{2}$ chemical engines in the magnetosphere for the SPS program. As we can see, the information available is not sufficient for a complete environmental assessment although it does allow limits of magnetospheric modifications to be roughly defined. The evolution of each exhaust emission outside of the immediate vicinity of the relevant spacecraft represents the effects of magnetospheric modification. These will be treated separately in the rest of this report. 


\section{ARGON ION ENGINE EXHAUST (COTV) IN MAGNETOSPHERE}

Argon plasma exhaust from the COTV ion engine assemly is the major source of magnetospheric effects; therefore, this section sets the foundation for major tasks in this assessment effort. A complete and quantitative description of the evolution of the injected argon plasma from the initial beam stage to the final thermalized and recombined stage is a task probably beyond present scientific capability. Yet, a satisfactory picture of the evolution can be obtained by breaking it down into a collection of important phenomena taking place at various magnetospheric regions and in various stages of argon plasma evolution. In this Section, we shall concentrate on the interactions between the $\mathrm{Ar}^{+}$exhaust and the magnetosphere as foundation for consideration of impacts in subsequent sections.

As is noted in Sections $\mathrm{II}$ and $\mathrm{II}$, the geometry of $\mathrm{Ar}^{+}$beam injection and the likelihood of $\mathrm{Ar}^{+}$precipitation into the atmosphere can be used roughly to divide the magnetospheric effects into two regimes. First, near LEO where the loss-cone is large a substantial portion of $3.5 \mathrm{keV} \mathrm{Ar}^{+}$is likely to precipitate as a dense beam, stimulating artificial local airglow and atmospheric heating some orders of magnitude above the natural aurora. Second, in the plasmasphere ( $\geq 2500 \mathrm{~km}$ altitude) and near GEO, the loss-cone becomes small and the $\mathrm{Ar}^{+}$beam is likely to undergo magnetospheric interactions as a plasma. In what follows we shall discuss the approximate behavior of the argon ion engine exhaust in these two regimes as groundwork for assessing the various specific magnetospheric effects.

By examination of (4), it is well-established that $3.5 \mathrm{keV} \mathrm{Ar}^{+}$emitted at LEO with nonvanishing $v_{\|}$is likely to charge exchange with neutral thermospheric constituents without first losing its initial energy by other interactions. A more quantitative consideration requires determination of the charge-exchange altitude $z_{c e}$ [or $r_{\ell}$ in (4)]. Since major thermospheric neutral constituents are $\mathrm{O}, \mathrm{N}_{2}$ and $\mathrm{O}_{2}$, the following chargeexchange processes must be considered:

$$
\begin{gathered}
\mathrm{Ar}^{+}+\mathrm{O} \rightarrow \mathrm{O}^{+}+\mathrm{Ar} \\
\mathrm{Ar}^{+}+\mathrm{N}_{2} \rightarrow \mathrm{N}_{2}^{+}+\mathrm{Ar} \quad\left[\sigma \simeq 1.2 \times 10^{-16} \mathrm{~cm}^{2}\right] \\
\mathrm{Ar}^{+}+\mathrm{O}_{2} \rightarrow \mathrm{O}_{2}^{+}+\mathrm{Ar} \quad\left[\sigma \simeq 4.5 \times 10^{-16} \mathrm{~cm}^{2}\right]
\end{gathered}
$$


These processes at $3.5 \mathrm{keV}$ incident energy are needed to determine $\mathrm{z}_{\text {ce }}$ for a given distribution of neutrals. The cross-sections associated with (6) and (7) were measured by Hedrick et al. [1977] but there is no known measurement of total cross-section for (5). If the incident energy far exceeds the $\mathrm{O}_{2}$ binding energy, we would expect that roughly

$$
\sigma[(5)] \simeq \sigma[(7)] / 2 \simeq 2.25 \times 10^{-16} \mathrm{~cm}^{2}
$$

With the use of the CIRA/COSPAR International Reference Atmosphere (1972) at an average $1000^{\circ} \mathrm{K}$ exospheric temperature, it is a simple numerical procedure to determine $z_{\text {ce }}$ from the functional relation (2) for each of the processes (5) - (7). We obtain:

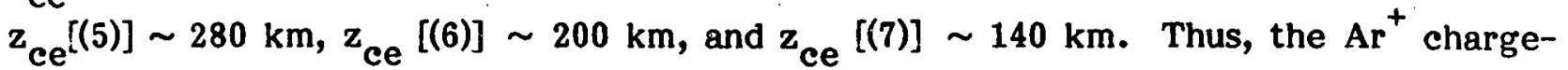
exchange altitude can be taken as $280 \mathrm{~km}$. This is due to charge exchange with atomic oxygen, which is the main neutral thermospheric constituent. With $r_{l}=R_{E}+280 \mathrm{~km}$, we can use (4) to calculate the loss-cone angle ( $\alpha_{\text {crit }}$ ) for give a field line $L \equiv R / R_{E}$ and for various locations of injection $r_{0}$ on the same field line. The relationship between $\alpha_{\text {crit }}$ and $r_{0}^{\prime}$ is shown in Figure 6 . From this figure, we determine that prompt precipitation of substantial numbers of $\mathrm{Ar}^{+}$of $3.5 \mathrm{keV}$ energy is unlikely for source radial distances greater than $\sim 2000 \mathrm{~km}$ from LEO, primarily because the beam pitch-angle is not likely. to be much less than $\sim 30^{\circ}$. Further consideration of precipitation of $3.5 \mathrm{keV}$ $\mathrm{Ar}^{+}$is given in Subsection V.A.

Thus, in the rest of the magnetosphere at radial distances $\sim 2000 \mathrm{~km}$ from LEO and up to GEO, trapping of the argon plasma, as depicted in Figure 2, is likely to occur for substantial length of time if the argon plasma beam does not pass entirely out of the magnetosphere. For this case, we must consider beam-plasma interactions. The plasma interactions can be roughly divided into the largest spatial scale interaction with the magnetospheric shell as a whole and into the spectrum of smaller scale plasma instabilities, although the two are not unrelated. Here we shall deal only with the former; discussion of the latter is found in Subsection V.E.

The physics of a plasma beam propagating transverse to a homogeneous vacuum magnetic field is very simple: if the beam is sufficiently dense so that polarization currents can maintain the charge separation electric field necessary to satisfy $\vec{E}+\frac{1}{c} \vec{v} \times$ $\vec{B}=0$ ( $\vec{v}$ is the beam velocity), the beam will propagate across the magnetic field. An alternative view of the effects of the polarization electric field $\vec{E}$ seen by a co-moving 


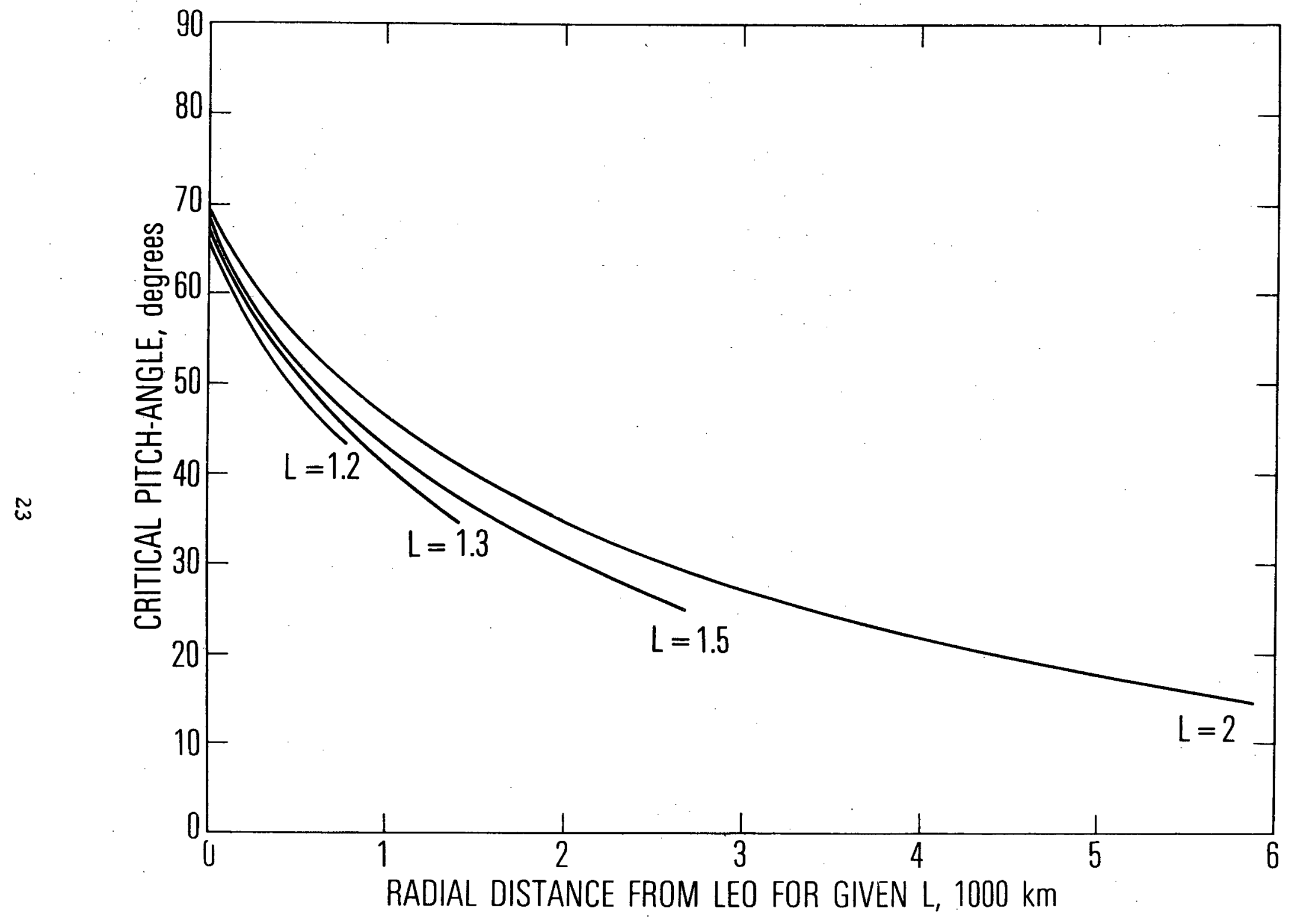

Figure 6. Critical Pitch Angle (Charge-Exchange Loss Cone Angle) Shown as Function of Radial Distance from LEO at Various L-Values. Loss cone narrows drastically for all $\mathrm{L}$-values at radial distances greater than $\sim 2000 \mathrm{~km}$ from LEO. 
observer above is that, in the coordinate system of the stationary magnetic field outside of the cloud, the plasma cloud, under the force of $\overrightarrow{\mathrm{E}}$, appears to be drifting with a velocity $\vec{v}=c(\vec{E} \times \vec{B}) / B^{2}$. But $\vec{v}$ above is also the drift velocity of magnetic field lines in the cloud induced by the electric field $\vec{E}$; hence, the field lines in the cloud are said to be "frozen" into the plasma, drif ting with velocity $\vec{v}$ relative to the field lines outside of the cloud. For this condition to apply, the beam density $n_{A}$ must satisfy

$$
4 \pi n_{A} m_{A} c^{2} / B^{2} \gg 1
$$

where $m_{A}$ is the argon-ion mass (Curtis and Grebowsky, 1979). Numerically, (9) yields (500-30000) $n_{A}\left(\mathrm{~cm}^{-3}\right) \gg 1$ for $2 \leq L \leq 4$, which would seem to be well-satisfied for the beam parameters of Table I; if so, the beam simply moves out of the magnetosphere to be dissipated in space.

Unfortunately, this conclusion is false. The magnetosphere, and particularly the ionosphere, cannot be considered in terms of a vacuum magnetic field. Plasmas in the magnetosphere, and especially in the ionosphere, act to short out the charge-separation electric field $\vec{E}$ and stop the beam in a distance of $\sim 1000 \mathrm{~km}$.

The basic physics of beam plasma cloud interaction with the magnetosphere and ionosphere is well-known, and was worked out by Scholer (1970) and Pilipp (1971) in connection with the HEOS release of an ionized barium cloud at $L=12$ (Haerendel and Lust, 1970). This high-altitude release had, as will the argon engines, a high initial $\beta$ (= $8 \pi \mathrm{P}_{1} / \mathrm{B}^{2}$, where $\mathrm{P}_{\perp}$ is the pressure perpendicular to the field lines of the injected plasma). The beam expands rapidly, in a direction perpendicular to $\vec{v}$ and to $\vec{B}$, to the point where $\beta \leq 1$. Of course, the beam also spreads without constraint (except for mirroring forces) along $\vec{B}$ as in Figure 2 and as discussed above.

One could calculate the final beam spread $\Delta y$ in the $y$-direction (or $\vec{v} \times \vec{B}$ direction; see Figure 2) using zero-Larmor-radius magnetohydrodynamics, that is, by equating $P_{1}$ (including thermal pressue $n_{A} k T$ plus dynamic pressure $1 / 2 n_{A} m_{A} \cdot v^{2} \tan ^{2} \theta$ ) to the asymptotic plasmaspheric pressure, which is essentially $B^{2} / 8 \pi$. Flux conservation requires $n_{A}=n_{0 A} A / \Delta z \Delta y$, where $n_{0 A}$ and $A$ are the initial beam density and area (see Table $\mathrm{I}$ ) and $\Delta \mathrm{z}$ is the beam spread along the field. Assuming $\Delta \mathrm{z} \geq \Delta \mathrm{y}$, one finds that $\Delta y$ is less than $\sim 1 \mathrm{~km}$ from $\mathrm{L}<4$, much smaller than the argon Larmor radius $R_{A}$. In effect, this calculation of the confinement of gyration centers tells us 
that $\Delta y$ is of the order of the argon Larmor radius $(40-80 \mathrm{~km}$ at $\mathrm{L}=4$ ) because the gyration centers are confined to a $\Delta y$ much smaller than the argon Larmor radius, at least for the first ten or so Larmor radii downstream from the nozzle; past this, the $10^{\circ}$ angular divergence of the beam could produce substantially larger $\Delta y$ [as long as (9) continues to hold].

In first approximation, then, we have a beam of $\Delta y \approx R_{A}$ propagating across the earth's field as shown in Figure 7. In this figure, the dotted lines show schematically earth's magnetic field lines at various times. The condition $\vec{E}+(1 / c) \vec{v} \times \vec{B}=0$ means that these lines are frozen into the plasma beam at the equator; their distortion is an Alfvén wave $(t=1$, in Figure 7 ). At $t=2$, the wave reaches the ionosphere, where the foot of the field line slips, because of the ionosphere's finite conductivity; the wave then reflects back to the beam $(t=3,4)$.

The field lines act somewhat like rubber bands, tending to retard the cloud. The physical mechanism is that the polarization charges responsible for $\vec{E}$ move along the field lines at the Alfvén speed $v_{A}$, accelerating magnetospheric plasma and transferring momentum out of the beam. Ultimately, the Alfvén wave reaches the ionosphere and drives dissipative Pedersen currents (in the absence of dissipation, the argon beam would oscillate like a mass on a rubber band field line).

Let $\mathrm{M}_{\mathrm{A}}$ be the mass density of the argon beam, integrated along field lines passing through the beam:

$$
\mathrm{M}_{\mathrm{A}}=\int \mathrm{dz} \mathrm{n}_{\mathrm{A}} \mathrm{m}_{\mathrm{A}}
$$

When this mass density is equal to the mass per unit area incorporated by the Alfvén wave, namely $2 v_{A} \tau n_{0} m_{p}$, the beam is essentially stopped. Here $\tau$ is the time it takes the Alfvén wave to travel a distance $v_{A} \tau$, and $n_{0} m_{p}$ is the magnetospheric mass density per unit volume. For the argon beam, $M_{A} \approx 5 \times 10^{-13} \mathrm{gm} \mathrm{cm}^{-2}$ at $L=4$; if $v_{A}$ $\approx 3 \times 10^{7} \mathrm{~cm} \mathrm{sec}^{-1}$ and $\mathrm{n}_{0} \approx 10^{3} \mathrm{~cm}^{-3}$, this gives $\tau \approx$ few seconds. The beam's velocity behaves like $v \simeq v_{0} e^{-t / \tau}$, so the beam can only travel a distance of the order of $v_{0} \tau \approx$ $10^{3} \mathrm{~km}$. In this example, the beam momentum is soaked up by magnetospheric plasma extending at most a few thousand kilometers down the field line on either side of the beam. 


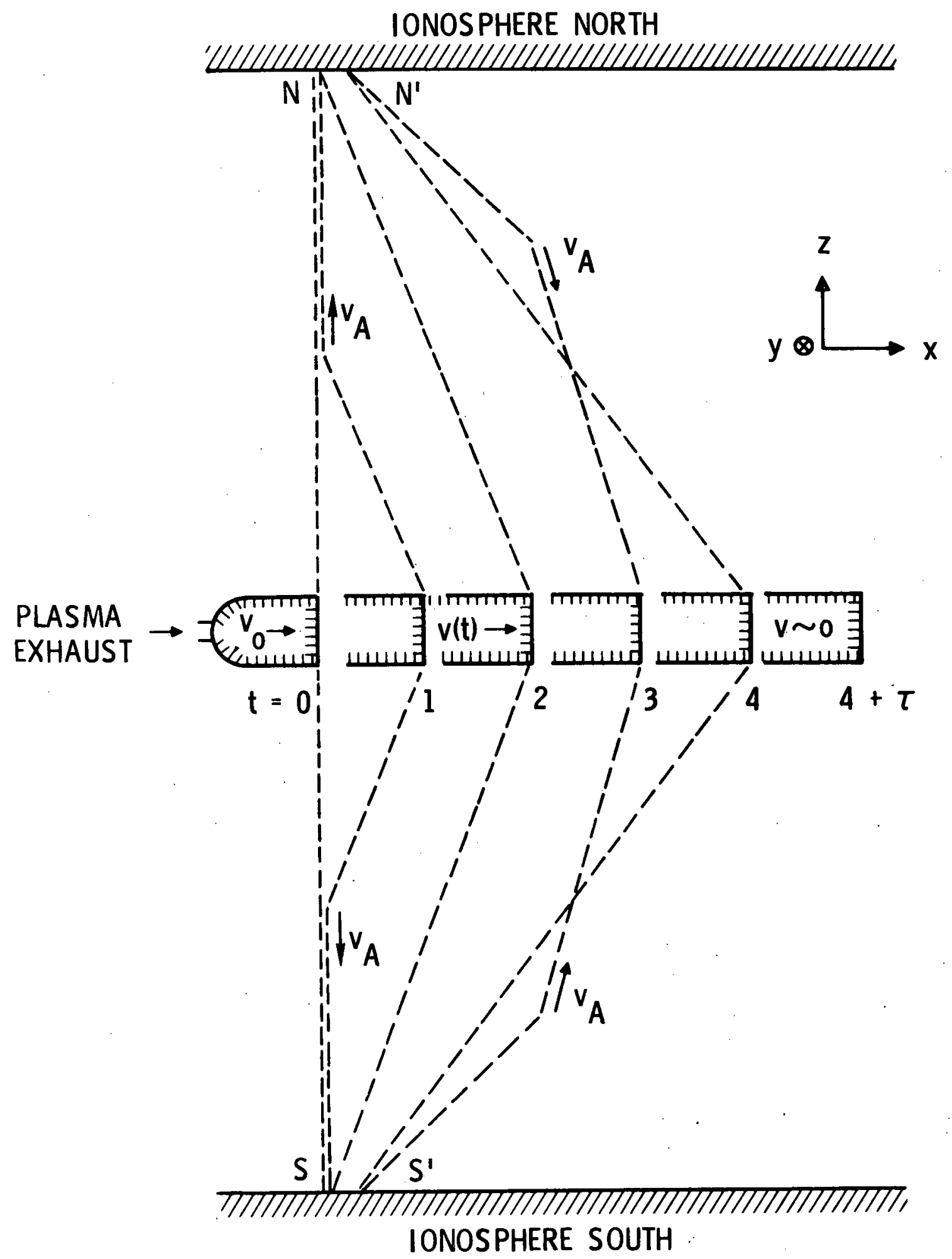

Figure 7. Schematic Representation of Beam-Magnetosphere and Beam-Ionsphere Interactions Involving Propagation and Reflection of an Alfvén Wave. Curvature of magnetic field lines and gradient of magnetic field intensity are not represented in this schematic model. 
This picture of argon beam plasma cloud interaction with the magnetosphere is based on high $\beta(\beta \gg 1)$ explosive barium releases in the far magnetosphere (Pilipp, 1971; Scholer, 1970). The $\beta$ of ion engine exhaust is much greater, but a second "confirmation" of the action of an Alfvén shock to short out the polarization electric field can be found in the stopping of Starfish debris motion perpendicular to the magnetic field. (Zinn et al., 1966), although for this case $\beta$ is again not as large as the case of the argon ion beam. It is sometimes claimed (Curtis and Grebowsky, 1979) that the argon ion beam regime is trans-Alfvénic, i.e., $v_{A} \leq v$, and that the physics of beam stopping is different because the beam speed is too fast for the Alfvén shock to act. However, actual calculation of plasmaspheric Alfvén speeds, as in Figure 8, based on a plasmasphere model which compares favorably with observations (Chiu et al., 1979b), shows conclusively that the $3.5 \mathrm{keV}$ argon ion beam is sub-Alfvénic by at least one order of magnitude. While the basic detailed physics of beam stopping requires further research, we feel that the physical ideas of the above references are sufficiently sound to allow us to estimate beam stopping characteristics. The basic physics of Alfvén shocks generated by a conducting body in the magnetosphere have also been treated (Drell et al., 1965).

The further fate of the injected $\mathrm{Ar}^{+}$after the major part of the initial $3.5 \mathrm{keV}$ energy has been imparted to the ambient magnetosphere may be very complex because it depends on the plasma dynamics of the $\mathrm{Ar}^{+}$cloud. We would expect a whole zoo of plasma instabilities to be operative; some of these may indeed hasten and spread the uniform beam stopping process discussed above. Some discussion of plasma instabilities are given in Subsection V.E. The longer-range evolution of $\mathrm{Ar}^{+}$is characterized by the competition between loss of energy by Coulomb collisions and the loss of $\mathrm{Ar}^{+}$itself by charge exchange. Long range Coulomb collisions with ambient electrons degrade the kinetic energy into ambient thermal energy without causing substantial pitch-angle change because these Coulomb collisions are primarily forward scatterings. For energetic $\mathrm{Ar}^{+}$to be physically lost from the magnetosphere, they will have to suffer charge exchange collisions which would allow the neutral argon atom to escape the magnetosphere. Figure 9 shows the comparison of charge exchange lifetimes of $\mathrm{Ar}^{+}$in the magnetosphere ( $\mathrm{L} \geq 2$ ) with the thermalization lifetime due to Coulomb collisions. In this Figure, the lifetimes are computed for lower energies because substantial fractions of the initial $3.5 \mathrm{keV}$ have been lost in the beam stopping process. Also, these lifetimes are computed for $\mathrm{Ar}^{+}$mirroring in the equatorial plane primarily because of 
the previous consideration that $\mathrm{Ar}^{+}$are unlikely to reach the loss-cone if they are injected at $>2000 \mathrm{~km}$ from LEO. The charge-exchange process in the magnetosphere is quite different from the processes (5), (6) and (7) because the magnetospheric neutral constituent is primarily hydrogen, i.e.,

$$
\mathrm{Ar}^{+}+\mathrm{H} \rightarrow \mathrm{H}^{+}+\mathrm{Ar}
$$

is dominant. The cross-section for (11) is unknown, but again for sufficiently high energy $\sigma[(11)] \simeq \frac{1}{2} \sigma\left[\mathrm{Ar}^{+}+\mathrm{H}_{2}\right]$. The $\mathrm{Ar}^{+}+\mathrm{H}_{2}$ charge-exchange cross-section has been measured by Gilbody and Hasted (1956). Note that the charge-exchange lifetimes $(\sim 100$ hrs) are comparable to the average duration between magnetosperic storms which typically defines the lifetimes of the natural magnetospheric plasma. Thus, we expect that the injected $\mathrm{Ar}^{+}$plasma will substantially modify the magnetosphere with a time constant roughly equal to several days until a new magnetospheric storm sweeps out the accumulation, if the storm mechanism remains effective under the modified circumstances. 


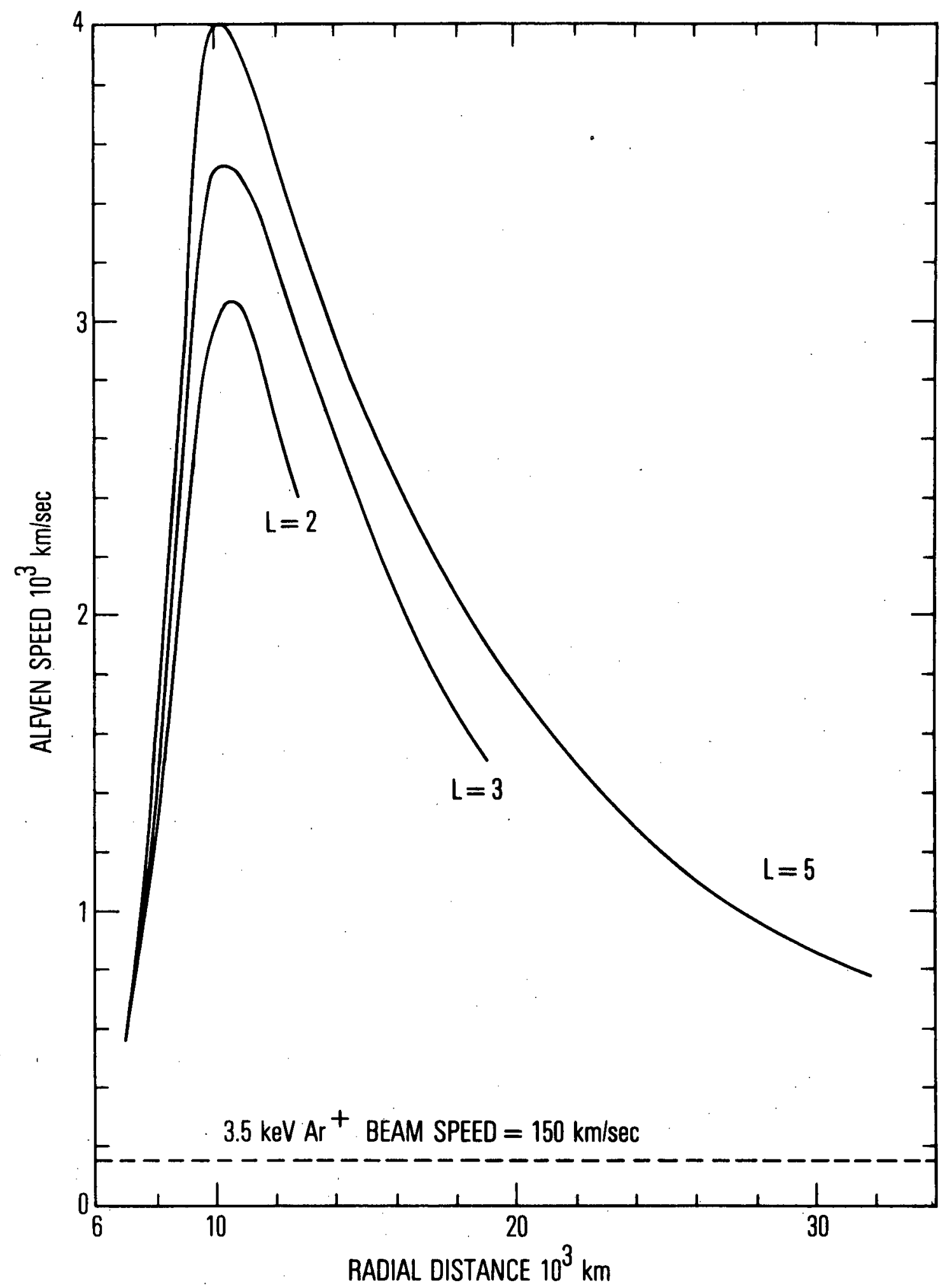

Figure 8. Alfvén Speed Shown as Function of Radial Distance from Earth's Surface for Geomagnetic Field Lines of Various L-Values: With the exception of a very local region near $500 \mathrm{~km}$ altitude, Alfve'n speed is well above the $3.5 \mathrm{keV}$ argon beam speed. Near LEO a substantial fraction of the beam may precipitate promptly. 


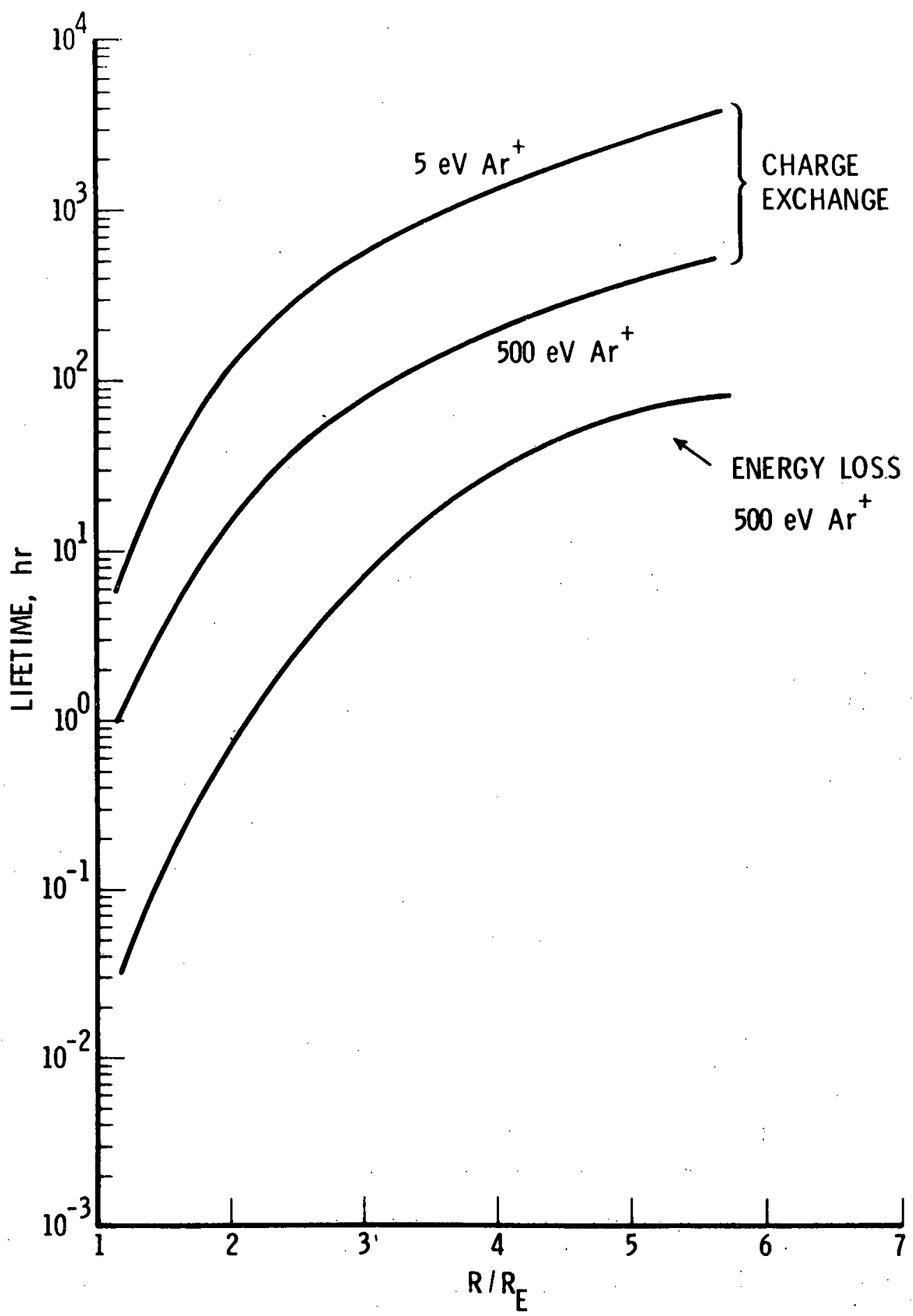

Figure 9. Comparison of Charge Exchange and Coulomb Lifetimes of $\mathrm{Ar}^{+}$Ions at Various Geocentric Distances in Equatorial Plane. Charge exchange cross section used in calculation of lifetime was measured by Gilbody and Hasted (1956). Note that $\mathrm{Ar}^{7}$ ions are assumed to have lower energies because of the beams stopping mechanism. 


\section{v. MAGNETOSPHERIC EFFECTS DUE TO ARGON PLASMA EXHAUST}

In this Section, we shall apply the physical interactions discussed in the previous Section to phenomena affecting human activities in space and on the ground. The entire Section is organized into five Subsections, although the Subsections are by no means independent.

\section{A. Artificial Airglow and Optical Earth-Sensor Interference}

Space-based optical sensors that peer at or through the earth's atmosphere for military, scientific, or earth-resource applications are widely used at the current time, and prognostications indicate even further exploitation of remote sensing techniques in the future. The interpretation of data from such systems must, of course, take into consideration the optical emissions that emanate from the atmosphere itself. For many if not most applications the atmospheric emissions represent an unavoidable background against which measurements must be made. Quite understandably, any changes in the background would have impact on the performance of such systems via their effect on data analysis and interpretation. In cases of extreme changes in the background sensor design modifications might be called for.

In the unperturbed low and mid-latitude regions of the upper atmosphere the optical emissions are called collectively "The Airglow". They range in wavelength from the extreme ultraviolet to the far infrared. These radiations, which are comprised mainly of lines and bands of $\mathrm{O}, \mathrm{O}_{2}, \mathrm{~N}_{2}$, NO and $\mathrm{H}$, are produced by various processes such as resonant and fluorescent scattering of sunlight, photoelectron excitation, and chemical interaction. It is instructive at this point to distinguish between photoelectrons in the ionosphere, which are the immediate product of the photoionization of neutral atmospheric particles, and the ambient electron gas, which is the ensemble of electrons that have reached thermal equilibrium. Photoelectrons are the more energetic of the two populations, capable even of producing further ionization, whereas the thermal electrons in the present undisturbed ionosphere have energies only of the order of a few tenths of an electron volt. In the auroral region, much more intense radiations of primarily the same character are stimulated by precipitated auroral electrons and ions with typically $1-30 \mathrm{keV}$ of energy; these are properly known as the aurorae.

The energy deposition associated with the argon ion engines of the SPS stimulates artificial airglow in two ways. First, the beam stopping mechanism heats the 
ambient magnetosphere and ionosphere (see IV, V.B., V.C. and V.E.) to a thermal energy level of a few electron volts, comparable to the energy of natural photoelectrons and therefore capable of stimulating similar airglow. This source of artificial airglow involves the natural atmospheric constituents and the well-known natural emission lines. The intensity is expected to be much greater than the natural airglow because the thermal electron flux is orders of magnitude higher than the photoelectron flux; however, it is not possible to assess at present exactly what the modified thermal energy level is, except that averaging consideration would put it in the $0.2-5 \mathrm{eV}$ range. The most important consequence appears to be the enhancement of the well-known atomic oxygen lines at $5577 \AA$ and $6300 \AA$. The ${ }^{1} S$ and ${ }^{1} D$ states of atomic oxygen, which lie at about 4 and $2 \mathrm{eV}$ respectively above the ground state and give rise to the aforementioned lines, can easily be excited by thermal electrons of $\sim 5 \mathrm{eV}$. Under natural conditions these lines originate from photoelectron impact and ion-chemical processes. In a similar context, thermalized argon ions may also modify oxygen and nitrogen airglow intensity because they react efficiently with $\mathrm{N}_{2}$ and $\mathrm{O}_{2}$ in charge exchanging processes:

$$
\begin{aligned}
& \mathrm{Ar}^{+}+\mathrm{N}_{2} \rightarrow \mathrm{Ar}+\mathrm{N}_{2}^{+} \\
& \mathrm{Ar}^{+}+\mathrm{O}_{2} \rightarrow \mathrm{Ar}+\mathrm{O}_{2}^{+}
\end{aligned}
$$

Process (12) is followed by

$$
\mathrm{N}_{2}^{+}+\mathrm{O} \rightarrow \mathrm{NO}^{+}+\mathrm{N}
$$

which is sufficiently exothermic to electronically excite either product. Process (13) is immediately followed by the dissociative recombination reacion

$$
\mathrm{O}_{2}^{+}+\mathrm{e} \rightarrow \mathrm{O}+\mathrm{O}
$$

in which one or both of the oxygen atoms may be excited and capable of radiating at either $5577 \AA$ and $6300 \AA$.

It is difficult to assess the impact of this source of airglow modification not only because we do not know the exact level of thermal modification without doing original research but also because the oxygen (red and green) regions of the spectrum do 
not seem to extensively interfere with present space systems. A test example can be cited in the case of SAR arcs (stable auroral red) which are stimulated by similar thermal processes at the atmospheric footprints of the plasmapause. Satellite measurements of electron temperature near the plasmapause indicate temperatures of $\sim 10^{40} \mathrm{~K}$ $(\sim 1 \mathrm{eV})$. As far as we can determine SAR arcs do not seem to impact optical earthsensor systems.

A second mechanism of airglow modification is much more serious since it affects directly near-UV portions of the spectrum where some critical sensors operate. This is due to direct impact upon the atmosphere by $3.5 \mathrm{keV} \mathrm{Ar}^{+}$beam from the vicinity of LEO. We call this process "artificial aurora" because it is a direct analog except that the excitation agent is $3.5 \mathrm{keV} \mathrm{Ar}^{+}$instead of (1-10) $\mathrm{keV}$ electrons from the aurora. Unlike the case of auroral excitation, excitation cross-sections for $\mathrm{Ar}^{+}$collisions with atmospheric atoms and molecules are not as well-measured as with energetic electrons. The only applicable measurement is reported by Liu and Broida (1970) for the excitation reaction

$$
\mathrm{Ar}^{+}+\mathrm{N}_{2} \rightarrow \mathrm{Ar}+\mathrm{N}_{2}^{+}\left(\mathrm{B}^{2} \Sigma_{\mathrm{u}}^{+}-\mathrm{X}^{2} \Sigma_{\mathrm{g}}^{+}\right)
$$

where the emission of the excited $\mathrm{N}_{2}^{+}$in the measured UV spectral range of $(2500-5000)$ $\AA$ have a cross-section $\sigma[(16)] \simeq 20 \times 10^{-18} \mathrm{~cm}^{2}$ for $900 \mathrm{eV} \mathrm{Ar}{ }^{+}$impact. In addition, the emission cross-section for the $3914 \AA$ line of $(16)$ is $\sigma[3914 \AA] \simeq 1 \times 10^{-18} \mathrm{~cm}^{2}$ [Thomas, 1972]. These measurements allow us to make a sample assessment of the factors controlling possible interference with optical earth sensors.

Consider the impact of $\mathrm{Ar}^{+}$(density $\mathrm{N}_{\mathrm{A}}$, speed $\mathrm{v}_{\|}$) upon atmospheric nitrogen of density $\mathrm{N}$ and scale height $\mathrm{H}$ with excitation cross-section $\sigma$. The emission intensity $\mathrm{I}$ from a $1-\mathrm{cm}^{2}$ column is given by

$$
I=N_{A} v_{\|} N \sigma H / 10^{6} \text { Rayleighs }
$$

where the quantities in the numerator of the right hand side are expressed in cgs units. Thus the emission intensity depends entirely on the density distribution $N_{A}$, which depends in turn on the dynamics of the ion engine beam at LEO. Considerations of pitchangle distributions such as in Section II indicate that the magnetic field exerts little influence on the beam density distribution between LEO $(\sim 400 \mathrm{~km})$ and the charge 
exchange altitude $(\sim 300 \mathrm{~km})$; therefore, to study the mapping of beam density from the nozzle at LEO to the airglow-emission altitude, we need only solve the collisionless Boltzmann equation for the free expansion of a neutral gas with distribution function $f(\vec{v}$, $\vec{r}, t)$ for a source distribution $S(\vec{v}, \vec{r}, t)$ which is a jet.

$$
\frac{\partial f}{\partial t}+\vec{v} \cdot \nabla f=s
$$

Our source is a time-independent beam at the origin firing off $\mathrm{Ar}^{+}$with speed $v_{0}$ in the $z$-direction of a cylindrical coordinate system $(r, z, \phi)$. A cylindrical system in velocity space $\left(v_{r}, v_{z}, \lambda\right)$ is also used. The velocity distribution in the $r-$ direction is assumed to be a Gaussian with a cut-off constant $\bar{v}_{\mathbf{r}}$. We shall study the effect of this beam spread constant $\bar{v}_{\mathbf{r}}$ upon the artificial auroral emission intensity. A properly normalized (to the $\mathrm{Ar}^{+}$propellant flow rate $\mathrm{F}$ ) source function is

$$
S=\left(F / 2 \pi^{2} \bar{v}_{r}^{2}\right)(1 / r) \delta(r) \delta(z) \delta\left(v_{z}-v_{0}\right) \exp \left(-v_{r}^{2} / \bar{v}_{r}^{2}\right)
$$

where $\mathrm{F}\left(\mathrm{Ar}^{+} / \mathrm{sec}\right)$ is $\mathrm{F} \equiv \int \mathrm{d}^{3} \mathrm{r} \mathrm{d}^{3} \mathrm{vS}$. The exact solution of (18) for $\mathrm{S}$ given by (19) can be obtained as

$$
f(\vec{r}, \vec{v}, t)=\int_{0}^{t} d t^{\prime} \int d^{3} r^{\prime} \delta\left[\vec{r}-\vec{r}^{\prime}-\vec{v}\left(t-t^{\prime}\right)\right] s\left(\overrightarrow{r^{\prime}}, \vec{v}\right)
$$

The density $\mathrm{N}_{A}$ is given by

$$
N_{A} \equiv \int d^{3} v f(\vec{r}, \vec{v}, t)=\left(F v_{0} / \pi \bar{v}_{r}^{2} z^{2}\right) \exp -\left(r v_{0} / z \bar{v}_{r}\right)^{2}
$$

From (21), we note that the beam density falls off as $\mathrm{z}^{-2}$ in the beam propagation direction but more importantly the beam is confined to a transverse region $r \leq z \bar{v}_{\mathbf{r}} / \mathbf{v}_{0}$. Since $z$ and $v_{0}$ are given for our case, the constant $\bar{v}_{\mathbf{r}}$ determines the cross-sectional area of the beam. The magnitude of $\bar{v}_{\mathbf{r}}$ is bounded by two limiting cases. For Case 1 , a rather unrealistic case; all particles make a $10^{\circ}$ angle with the nozzle axis as they emerge (including those from the middle of the nozzle); we have $\bar{v}_{\mathbf{r}} / \mathbf{v}_{0}=\tan 10^{\circ}$. From (21), it is seen that Case 1 yields a beam which spreads very fast. For Case 2, the perpendicular spread of the beam is due entirely to thermal motion, a more realistic case for an exhaust jet; then, $\bar{v}_{\mathrm{r}}=\left(2 \mathrm{kT} / \mathrm{m}_{\mathrm{A}}\right)^{1 / 2}$ where $\mathrm{T}$ is the $\mathrm{Ar}^{+}$temperature $\left(\sim 1000^{\circ} \mathrm{K}\right)$. This yields a dense beam. The truth probably lies between these two limits. 
Table II. Artificial and Natural $\mathrm{N}_{2}^{+}(2500-5500 \AA)$ A Aroral Intensities.

Altitude

350

300

$250^{*}$

200

$150^{* *}$
$\underline{I}_{1} \underline{\text { (rayleighs) }}$

1.24

1.30

2.50

6.5

24.4
$\underline{I_{2}} \underline{\text { (rayleighs) }}$

$1.58 \times 10^{6}$

$1.65 \times 10^{6}$

$3.17 \times 10^{6}$

$8.25 \times 10^{6}$

$3.1 \times 10^{7}$
Natural (rayleighs)

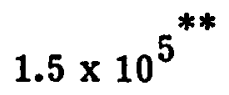

${ }^{*} \mathrm{Ar}^{+}+\mathrm{O}$ charge exchange altitude. Below this altitude, the $\mathrm{Ar}^{+}$become fast neutrals and the calculated intensities are not accurate.

**Electrons of energy (1-10) keV penetrates much deeper than $\mathrm{Ar}^{+}$of $3.5 \mathrm{keV}$ and generates aurora in this region and below. 
We have computed the column emission intensity for these two cases $\left(I_{1}\right.$ and $\mathrm{I}_{2}$ ) using (21) and (17) for propellant flow rate given in Table I and for a CIRA (1972) atmosphere with $1000^{\circ} \mathrm{K}$ exospheric temperature. The artificial $\mathrm{N}_{2}^{+}$aurora brightness stimulated by $3.5 \mathrm{keV} \mathrm{Ar}^{+}$in the spectral range 2500-5500 $\AA$ range is given in Table II. For comparison, the brightness of the natural aurora of moderate strength emitted by the same $\mathrm{N}_{2}^{+}$band in the 3500-5500 $\AA$ range (Baker and Pendleton, 1976) is also listed. It is seen that the artificial auroral may be an order of magnitude brighter in intensity than the natural aurora in these spectral bands. It must be noted, however, that this result applies only to $\mathrm{Ar}^{+}$beams emitted at LEO. Since these spectral regions may be of importance to optical earth-sensors, our considerations suggest that such interference can be mitigated by a thrusting schedule which directs the $\mathrm{Ar}^{+}$beam at LEO primarily in the azimuthal direction (perpendicular to the magnetic field) as in Figure 2 until the COTV is more than $\sim 2000 \mathrm{~km}$ from LEO. This thrusting schedule would require orbit plane change operations to be performed in the magnetosphere rather than at LEO. This is an interesting case in which environmental effects may feed back upon the system design concept.

\section{B. Ionospheric and Plasmaspheric Heating}

In this Subsection, we move away from LEO and consider the prompt effects of the interaction between the $\mathrm{Ar}^{+}$beam and the magnetosphere as described in Section IV and Figure 7. We recall that the $\mathrm{Ar}^{+}$beam exchanges energy with the entire ambient magnetosphere via the propagation of Alfvén shock waves before the $\mathrm{Ar}^{+}$particles are physically present in the entire magnetosphere.

The ionosphere "feels" the beam after a time $\approx \ell / v_{A} \approx$ tens of seconds $(\ell$ is the length of the field line and $v_{A}$ is the Alfvén speed). The electric field $E_{I}$ imposed on the ionosphere differs from the $\frac{1}{c} \vec{v} \times \vec{B}$ field, mapped to the ionosphere, for two. reasons: first, the electric field diminishes along the field line by a factor of order exp $\left(-\ell / v_{A} \tau\right)$, and second, the electric field is partly reflected at the ionosphere (to produce the upgoing Alfvén wave at $t=3,4$ in Figure 7). Scholer (1970) gives the relation

$$
\begin{aligned}
E_{I} & =[2 /(1+x)] E_{o u t} \\
x & =4 \pi \Sigma_{p} v_{A} / c^{2}
\end{aligned}
$$


where $\mathrm{E}_{\text {out }}$ is the field just outside the ionosphere, $\mathrm{E}_{\mathrm{I}}$ the field in the ionosphere, and $\Sigma_{\mathrm{p}}$ the ionospheric Pedersen conductivity. It is somewhat hazardous to make numerical estimates with exponential factors floating around, but it would be surprising if the ionospheric time scale for dissipation, $\tau_{I}$, defined by

$$
\tau_{I}=m_{A} v_{0}^{2} / 2 \Sigma_{p} E_{I}^{2}
$$

were very much greater than $\tau$. In (24) $\mathrm{m}_{\mathrm{A}}$ is the argon mass and $\mathrm{v}_{0}$ is the argon speed. Taking the two time scales equal, the rate at which beam energy is dissipated in the ionosphere is roughly $\mathrm{m}_{\mathrm{A}} \mathrm{v}_{0}{ }^{2} / 2 \tau \approx \mathrm{few} \mathrm{ergs} / \mathrm{cm}^{2} \mathrm{sec}$, and this might occur over an area of $10^{3}-10^{4} \mathrm{~km}^{2}$ in the ionosphere.

Before we discuss the consequence of Joule dissipation of the beam-induced ionospheric electric field $\mathrm{E}_{\mathbf{I}}$, we note that it drives a Pedersen current across the magnetic field in the ionosphere in exactly the same manner as the auroral ionospheric current driven by the increased convection electric field. This ionospheric current appears, however, at the footprint of the magnetic field line connecting the COTV spacecraft and the ionosphere, i.e., at mid-latitudes. The natural auroral currents are known to induce relay-tripping effects in high latitude power transmission lines and to cause increased corrosive effects in long distance pipelines near the auroral zone (U.S. NOAA, 1980). Exactly what the effects of the artificial ionospheric current in the densely populated mid-latitude zones would be requires in-depth assessment of the transmission of such an artificial electric field through the mid-latitude ionosphere. The presence of such an ionospheric current necessarily implies Joule dissipation and consequent heating of the ionosphere. We argue that the major part of the beam power of $\sim 5 \times 10^{15} \mathrm{ergs} / \mathrm{sec}$ will be dissipated in the magnetosphere and ionosphere.

By comparison, a natural geomagnetic storm dissipates energy at a rate $10^{17} \mathrm{ergs} / \mathrm{sec}$, but only sporadically and over a much larger area. The dissipation rate per unit area for a storm is also a few ergs/ $\mathrm{cm}^{2}$ sec (Ching and Chiu, 1973), comparable to the argon beam dissipation. The difference is that the argon beam will be present, day and night, for several months, while storms occur only every few days. The amount of naturally-occurring energy precipitated into the ionosphere over a span of time like four months (the ion-engine operational period), is not likely to exceed a few times $10^{22}$ ergs per month in moderately quiet times; this is of the order of magnitude of the energy released into the plasmasphere by the SPS ion engines. 
Thus, the beam may well deposit (by Joule heating) a substantial amount (

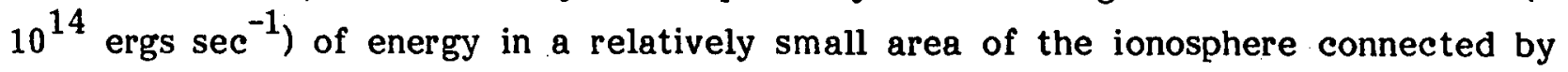
magnetic field line to the argon beam at any given moment of operation (see Figure 2), long before the majority of $\mathrm{Ar}^{+}$ions are physically present in the ionosphere. Since the COTV spirals outwards toward GEO, as in Figure 3, the relatively localized "footprint" of the instantaneous field line becomes a band of heated ionospheric-plasmaspheric flux shells. The exact level of heating and the exact extent of heated areas need to be evaluated for a complete assessment, but these can only be arrived at by doing applied research. For the present effort, it is perhaps sufficient to seize upon the similarity in magnitude between beam energy dissipation and geomagnetic storm energy dissipation in the ionosphere; and note that the heating input is not unlike that in a flux tube containing the auroral arc. It has been estimated that in the auroral arc approximately one-half of the heating is by particle dissipation and the other half by Joule dissipation (Evans/NOAA, private communication 1979). Since it is difficult to see how Joule dissipation of the electric field $E_{I}$ can be any different from Joule dissipation of the auroral electric field, we would expect the plasma conditions to be similar in the two flux tubes also. If so, then we would expect the plasma temperature to be a few electron volts $\left(10^{3}-10^{4} \mathrm{~K}\right)$. This estimate is also consistent with the averaging approach taken in Section IV and Figure 9.

What is the primary consequence of ionospheric-plasmaspheric heating to a few electron volts?. We argue that this will lead to a modification of plasmaspheric composition. This argument is based on a steady state model of the density and composition distribution of the plasmasphere; thus, it needs qualification and refinement based on further applied research. However, since the results are physically sound, we feel that the first order estimates are sufficiently significant for present purposes.

As a first approximation it is assumed that the ionized argon can be treated as a constituent of the steady-state plasmasphere. An earlier analytical model of the equilibrium plasmasphere derived by Chiu et al. (1979b) is used to predict the changes that will occur due to the presence of argon ions and to the heating of the ambient plasma by the thermalizaton of the argon ions.

Chiu et al. (1979b) found that the observed plasmaspheric density included in the region between an altitude of $500 \mathrm{~km}$ and the dipole field line $\mathrm{L}=5$ can be adequately described by the expression 


$$
\begin{gathered}
n_{j}(s)=n_{j}(\ell)\left[B(s) T_{j}(\ell) / B(\ell) T_{j}(s)\right] \cdot \exp \int_{\ell}^{s} d s^{\prime} \\
{\left[q_{j}|e| E-m_{j}\left(G M_{E^{\prime}} / r^{2}+\Omega_{E}{ }^{2} r\right) \hat{r^{\prime}} \cdot \hat{s}^{\prime}\right] / k T_{j}\left(s^{\prime}\right)}
\end{gathered}
$$

where $n_{j}$ is the density of ion species $j, s$ is the distance along the dipole field line measured from the equator $(s=0)$ to the ionosphere $(s=\ell), B$ is the magnetic field strength, $q \mid e l$ is the charge, $E$ is the ambipolar diffusion electric field strength, $m$ is the ion mass, $G$ is the gravitational constant, $M_{E}$ is the mass of the earth, $\Omega_{E}$ is the angular rotation frequency of the earth, $k$ is Boltzmann's constant, and $r$ is the geocentric radial coordinate. The empirically determined temperature profile along a field line is given by

$$
\mathrm{T}(\mathrm{s})=\mathrm{T}_{0}+\mathrm{T}_{1}\left[\frac{\mathrm{L}-1}{\mathrm{~L}_{0}}\right]^{\alpha}\left[\frac{\ell-\mathrm{s}}{\ell}\right]^{\beta}
$$

where $\mathrm{T}_{0}, \mathrm{~T}_{1}, \mathrm{~L}_{0}, \alpha$ and $\beta$ are constants with the following norminal values: $\mathrm{T}_{0}=$ $2000^{\circ} \mathrm{K}, \mathrm{T}_{1}=3000^{\circ} \mathrm{K}, \mathrm{L}_{0}=3, \alpha=1 / 2, \beta=1 / 2$. These authors considered a chargeneutral four constituent model composed of electrons, $\mathrm{H}^{+}, \mathrm{O}^{+}$and $\mathrm{He}^{+}$. Their result in the equatorial plane and the above natural temperature parameters is shown in the left panel of Figure 10.

For the purpose of the present investigation, the minor constituent $\mathrm{He}^{+}$was omitted and argon ions were substituted as the fourth plasmasphere constituent in the model. As mentioned above, the ionospheric $(500 \mathrm{~km})$ concentration of argon ions was set equal to either the local concentration of hydrogen ions or of oxygen ions as nominal lower and upper limits. It must be recognized that these limits are at present arbitrary; eventually, these limits should be set by a detailed study of the fate of $\mathrm{Ar}^{+}$in the magnetosphere. Several different fractional temperature increases were assumed. The results of the calculations are a bit tedious to be shown here in detail but the salient features are summarized by the right hand panel of Figure 10, in which $\mathrm{T}_{0}$ is set at $5 \mathrm{eV}$ and $T_{1}=0$. Our results show that the addition of the argon ions will not upset the normal distribution of the plasmaspheric hydrogen and oxygen; however, as shown by the right-hand panel, the addition of heat by the argon-ion interaction with the ambient medium or by Joule heating can drastically alter the plasmasphere. At high altitudes, hydrogen can be replaced by the heavier constituents (oxygen or a combination of oxygen and argon). These results are easily understood, because the increased heating drives $\mathrm{O}^{+}$ 
and $\mathrm{H}^{+}$up the field line but the supply of ionospheric $\mathrm{O}^{+}$is orders of magnitude higher than $\mathrm{H}^{+}$, thus increasing the proportion of $\mathrm{O}^{+}$in the higher altitude region. This situation is further accentuated by the gravitational sinking of $\mathrm{Ar}^{+}$relative to the accompanying electron, driving up more $\mathrm{O}^{+}$to maintain charge neutrality. It must be recognized also that the assumed boundary conditions are simplifications of the actual situation, which is very far from steady state. The argon ions will be introduced onto different $L$ shells at different times; plasma convection processes and radial diffusion can modify the spatial distribution of the argon which, because of the nature of its source, is already quite different from that of a normal plasmasphere constituent. In addition, interhemispheric flows, which may affect the plasmasphere as a whole were not included in the model. Work on including these effects is currently underway.

The consequence of having large concentrations of heavy ions in the plasmasphere can be quite serious for the dynamics of the radiation belt relativistic electrons. These effects will be discussed in Subsection V.C. and V.D.

\section{Ring Current Modifications}

Consideration of magnetospheric effects caused by the presence of $\mathrm{Ar}^{+}$ions may best be ordered in terms of the energies of various plasma constituents in the magnetosphere. This classification is particularly convenient because the beam-stopping mechanism slows down significant numbers of $\mathrm{Ar}^{+}$to ambient plasma energies ( $\left.\lesssim 1 \mathrm{eV}\right)$ while other $3.5 \mathrm{keV} \mathrm{Ar}^{+}$may escape uniform deceleration due to pitch-angle scattering or other smaller scale interactions.

For natural magnetospheric particles other than thermal particles, it is customary, for both kinematical and dynamical reasons, to distinguish between particles having $E \sim(1-100) \mathrm{keV}$ and those having $E \gtrsim 100 \mathrm{keV}$. The former are known as ringcurrent particles, since they account for the major share of diamagnetic disturbances observable with low-latitude magnetometers at the earth's surface. The latter are known as radiation-belt particles, since they account for most of the radiation damage to hardened spacecraft. The ring current and the radiation belts consist of electrons, protons, and heavier ions trapped in the earth's magnetic field. Such particles obey the laws of adiabatic charged-particle motion in the first approximation, and their spatial iso-intensity profiles are toroidal in shape (e.g., White, 1966).

The ring current and radiation belts are populated with energetic particles by virtue of the dynamical interaction between the earth's magnetosphere and the solar 


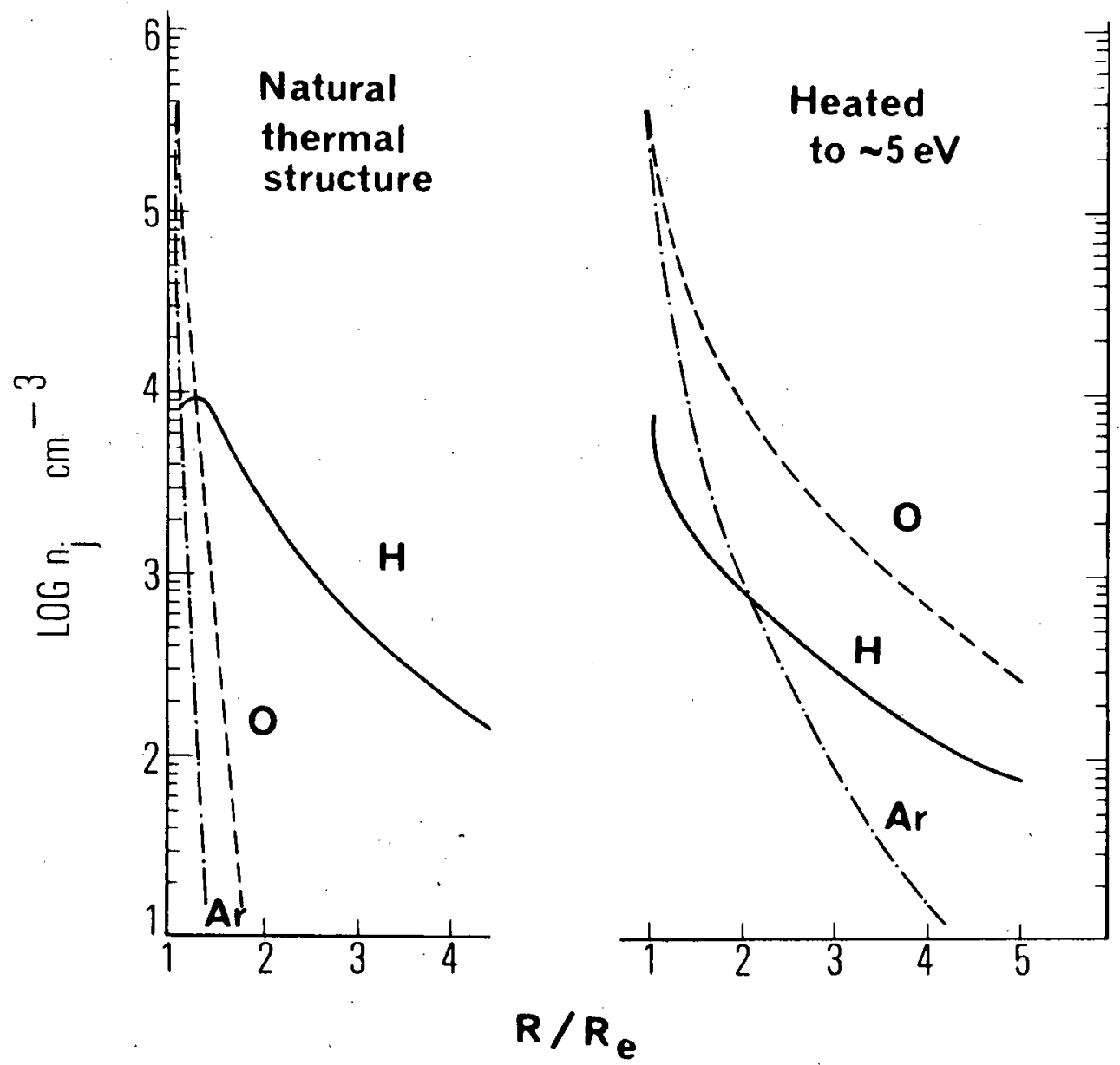

Figure 10. Comparis on of Compositional Structure of Plasmasphere Under Natural Thermal Conditions (Left Panel) and Under Heated, Circumstances (Right Panel). Natural $\mathrm{H}^{+}$ content at $500 \mathrm{~km}$ altitude level is assumed to be unaltered. Ionospheric release of $\mathrm{H}_{2} \mathrm{O}$ may alter this assumption but in any case substantial fluxes of $\mathrm{O}^{+}$are driven up to higher altitudes. 
wind. They are depleted of such particles mainly by interaction with plasma turbulence, which tends to violate the adiabatic invariants of charged-particle motion. Such violation allows particles to escape confinement by the earth's magnetic mirror and to precipitate (i.e., deposit their energy) in the earth's atmosphere and ionosphere. However, the interaction of geomagnetically trapped particles with plasma turbulence is very selective, in that it is contingent upon a "resonance" between the gyration of a particle and the frequency of a wave in the turbulent spectrum (after one takes account of the Doppler shift associated with the motion of the particle through the plasma). Thus, different classes of turbulence are found to interact with different classes of particles.

The energy spectrum of trapped $\mathrm{Ar}^{+}$ions in the magnetosphere may range from $3.5 \mathrm{keV}$ on down to a few eV. Thus they are properly classified as ring currents and thermal particles. In this Subsection, we shall discuss some features of their interactions while the systems effects of these interactions will be discussed in Subsections V.D. and V.E.

In order to discuss the main dynamical effects of heavy ion additions to the natural ring current (thermalized $\mathrm{Ar}^{+}$discussed above and thermal $\mathrm{O}^{+}$driven up from the ionosphere, as discussed in Subsection V.B.), we must discuss the physics of electromagnetic cyclotron instability in the magnetosphere, although the main discussion on the zoo of plasma instabilities connected with $\mathrm{Ar}^{+}$injection is in Subsection V.E.

Turbulence in a plasma can be created by various mechanisms. A main source of instability in the magnetospheric plasma is the velocity-space anisotropy intrinsic to ring-current particle distributions. Anisotropy here means that relatively more particles have larger velocity components parallel or perpendicular to the magnetic field. Thus iso-intensity contours in $\left(v_{\|}, v_{\perp}\right)$ - space are no longer circular. Since the magnetic mirror points of ring-current protons and electrons are concentrated near the magnetic equator rather than being distributed uniformly along a field line, there must be relatively more energy per degree of freedom associated with particle gyration than with translation of guiding centers along field lines. This condition can easily lead to instability in certain electromagnetic wave modes. The anisotropy of ring-current electrons can lead to instability of a field-guided wave with right-handed polarization, i.e., the so-called "whistler" wave mode. The anisotropy of ring-current ions can lead to instability of the analogous field-guided wave with left-handed polarization. Both 
instabilities require the wave frequency to be somewhat smaller than the corresponding particle gyrofrequency. Both instabilities cause velocity-space diffusion so as to reduce the anisotropy of the corresponding charged-particle species, and so as to reduce the lifetime of that species against precipitation into the earth's atmosphere (Kennel and Petschek, 1966; Cornwall, 1966, Cornwall et al., 1970). Moreover, the unstable ioncyclotron waves generated by the anisotropy of ring-current protons are resonant with relativistic radiation-belt electrons $(\mathrm{E} \gtrsim 2 \mathrm{MeV}$ ) and thus account for the observed precipitation of such electrons during the recovery phase of a magnetic storm (Thorne and Kennel, 1971; Vampola, 1971). It happens that the electromagnetic instabilities noted here are not effective at ring-current energies for protons outside the plasmasphere, since the larger phase velocities attained there require a correspondingly larger proton energy for cyclotron resonance. Thus, the precipitation of relativistic electrons is contingent on the spatial co-existence of ring current and plasmasphere, which occurs only during the plasmaspheric expansion characteristic of the recovery phase of a magnetic storm.

The electromagnetic proton-cyclotron instability, however, is likely to be suppressed by the presence of substantial numbers of heavy ions such as $\mathrm{Ar}^{+}$or $\mathrm{O}^{+}$in the magnetospheric plasma (Cornwall and Schulz, 1971). This means that the major mechanism for the depletion of relativistic electrons from the outer radiation belt is likely to be made inoperative by SPS ion engine injections of $\mathrm{Ar}^{+}$, resulting in enhancement of relativistic electron flux. Discussion of radiation belt relativistic electron effects is found in Subsection V.D.

There is no danger that the population of ring-current protons would be similarly enhanced, since these are subject to rapid charge exchange with ambient neutral hydrogen, as are ring-current helium and oxygen ions on a substantially longer time scale (Tinsley, 1976; Lyons and Evans, 1976). Moreover, the addition of argon plasma (or any other type of plasma) to the natural plasmasphere would tend to enhance the loss rate of ring-current electrons (Brice, 1970; 1971). One should not worry about the development of a charge imbalance in the magnetosphere on account of modified precipitation rates. There is ample cold plasma in the plasmasphere to balance charges through minor modifications of the already weak ambipolar electric field, which has been included in the model calculations in the previous section.

Suppression of the electromagnetic ion-cyclotron wave mode is illustrated in Figure 11. The normalized growth rate $\gamma / \Omega_{\mathrm{p}}$ is plotted as a function of normalized wave 


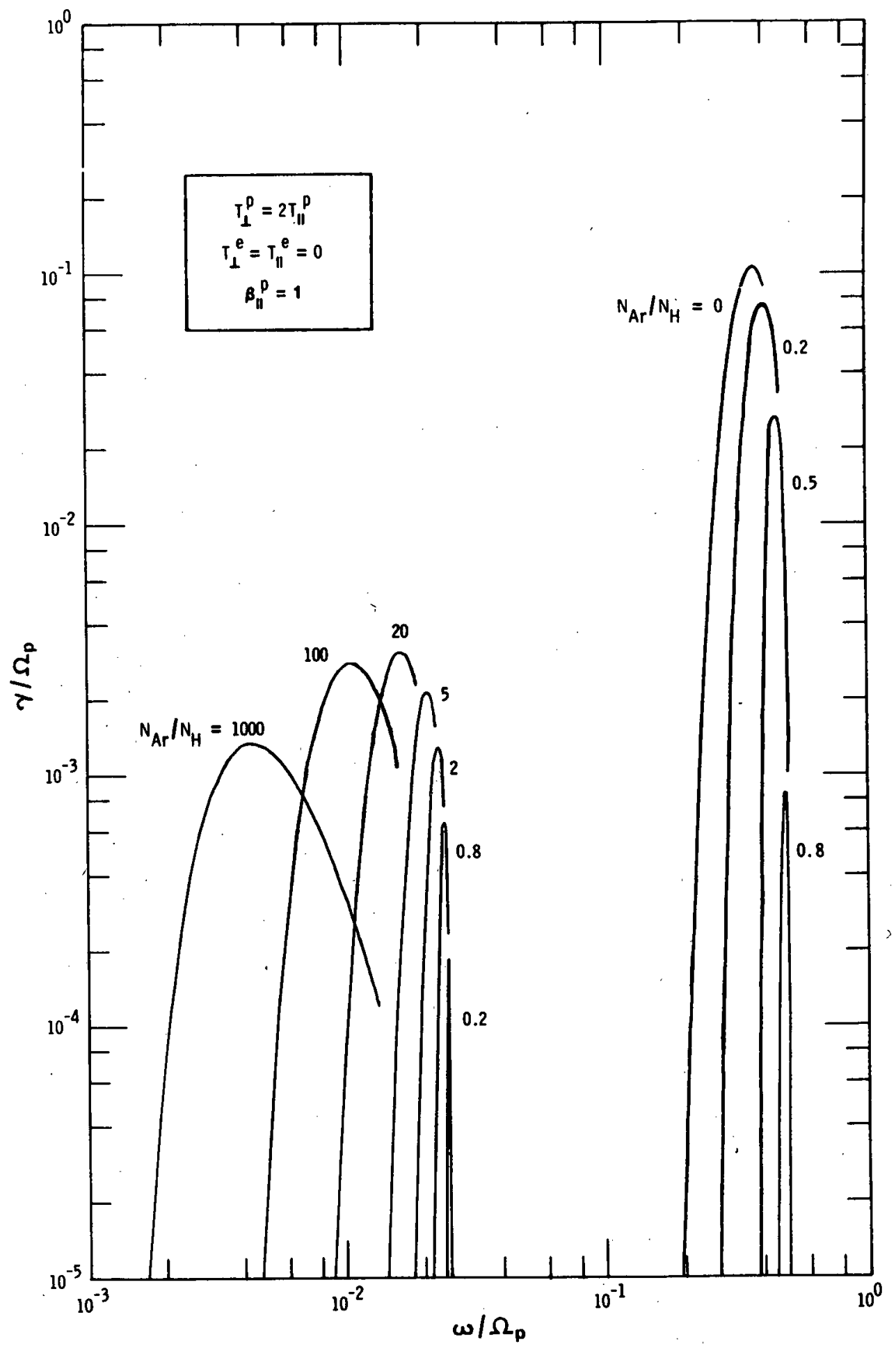

Figure 11. Lowest Order Estimates for Normalized Growth Rate as Function of Normalized Wave Frequency, for Various Relative Concentrations $\left(\mathrm{N} / \mathrm{N}_{\mathrm{H}}\right)$ of Argon and Hydrogen Plasma. Method of calculation is that of Märk (1974). 
frequency $\omega / \Omega_{\mathrm{p}}$ for selected argon/hydrogen plasma-concentration ratios. For the parameters chosen it is clear that a ratio $\mathrm{N}_{\mathrm{Ar}} / \mathrm{N}_{\mathrm{H}} \gtrsim 1$ is sufficient to suppress the proton-cyclotron branch altogether. The lower-frequency argon-cyclotron branch achieves a growth rate of at most 3 of the maximum proton-cyclotron growth rate attained in the absence of argon. Thus, the electromagnetic ion-cyclotron mode is very much stabilized overall by the addition of cold argon plasma to the medium. This conclusion is consistent with the results obtained by similar methods for the addition of even lighter ions such as $\mathrm{Li}^{+}$and $\mathrm{He}^{+}$(Märk, 1974).

On the other hand the injected 3.5-keV $\mathrm{Ar}^{+}$ions might be scattered out of the beam by some mechanism and come to constitute a plasma having a 3.5-keV temperature. In this latter case the injected argon plasma would contribute directly to the ring-current population, and the $\mathrm{O}^{+}$drawn up from the F-region would constitute the cold-plasma additive. The cold $\mathrm{O}^{+}$would tend to suppress the electromagnetic protoncyclotron and helium-cyclotron instabilities of the natural ring current at frequencies below the oxygen gyrofrequency (e.g., Cornwall and Schulz, 1971). However, the hot argon plasma would become anisotropic with time because of charge exchange, and would thus constitute an additional source of free energy for instability (cf. Cornwall, 1977). The argon-cyclotron instability would occur at frequencies somewhat below the argon gyrofrequency. Its growth rate would be enhanced by the presence of cold $\mathrm{O}^{+}$in the plasmasphere (cf. Cornwall and Schulz, 1971). The argon-cyclotron waves would tend to resonate wth relativistic electrons (cf. Thorne and Kennel, 1971) and thus to facilitate the loss of such trapped electrons into the atmosphere.

These energetic $\mathrm{Ar}^{+}$begin to act like a man-made ring current of $\gtrsim 1 \mathrm{keV}$ ions, with a residual pitch-angle anisotropy left over from the initial injection nearly perpendicular to B. Just what energy and anisotropy are left after the beam-plasma interactions is not known at present; they need to be evaluated in order to make a more complete assessment. This ring current acts much like a natural one, subject to charge exchange, Coulomb scattering (Section IV), and wave-particle interactions (see below). If charge exchange is the dominant loss process (Coulomb scattering and other forms of energy loss do not effectively remove argon), this ring current may have $\sim 10^{30}$ ions in it, comparable to the natural ring current. A major difference is that the argon ring current is mostly inside the plasmasphere, while the natural ring current penetrates perhaps one earth radius inside the plasmasphere with the rest outside. Nonetheless, there could be substantial overlap between the ring currents, especially during storm 
times when the plasmasphere is eroded and the natural ring current driven in by convection electric fields. The argon ring current can make a substantial contribution to the plasmaspheric pressure and hence to the currents which flow there (both across and along B); this will significantly stiffen the plasmasphere to deformations associated with enhanced storm-time convection electric fields and diminish the strength of electrostatic radial diffusion. The result may be (other things being unchanged) a buildup of ring-current particles and inhibition of inward transport of higher-energy particles.

In summary, SPS injection of $\mathrm{Ar}^{+}$and heating of the plasmasphere and ionosphere system necessarily imply substantial modification of the composition and density of the thermal and ring current particles of the magnetosphere. The increase of thermal heavy ion populations is likely to suppress ion cyclotron plasma turbulence, which is primarily responsible for scattering loss of radiation belt relativistic electrons. On the other hand, addition of thermal plasma would increase the loss rate of ring current electrons, thus modifying the auroral process during magnetic storms.

\section{Radiation Belt Modification}

The radiation belts consist of energetic electrons and ions $(E \gtrsim 100 \mathrm{keV})$ trapped in two toroidal shells in the magnetosphere (Figure 1a) earthward of GEO. The relativistic ion component probably originate from the decay of solar neutrons and from the effects of heavy cosmic-ray particles. The heavy particles, also known as HZE (for high atomic number and energy) particles, are the most penetrating and are hazardous to humans and to space-borne equipment. Fortunately, the flux of these HZE particles is low and is unlikely to be affected by artificial magnetospheric modification. Relativistic electrons, on the other hand, constitute a component as important as HZE particles in defining design limits to space systems. As is pointed out in the previous Subsection (V.C.), relativistic electrons are rather more likely to interact with magnetospheric turbulence by resonant pitch-angle scattering with ion cyclotron waves; therefore, ring current and thermal plasma modifications are likely to impact Van Allen belt relativistic electrons. The present population of such hazardous electrons is kept in balance by the occurrence of several large magnetic storms per year. During such large storms, relativistic electrons in the radiation belts are seen to precipitate into the atmosphere. Such an event is known as an REP (relativistic electron precipitation) event. Suppression of the proton-cyclotron instability might, therefore, result in a major enhancement of the radiation level from relativistic electrons within a year after argon-ion saturation of the plasmasphere. 
The enhancement of relativistic-electron radiation can be analyzed within the framework of a simple model. Consider the model equation (e.g., Schulz, 1974)

$$
\mathrm{dI} / \mathrm{dt}=\mathrm{S}-\lambda \mathrm{I}
$$

in which 1 represents the radiation intensity, $\lambda$ the natural radiation-belt decay rate, and $S$ the strength of a weak source of relativistic electrons. The decay rate $\lambda$ is perhaps inversely proportional to the particle energy $\mathrm{E}$, such that $1 / \lambda \approx(\mathrm{E} / 1 \mathrm{MeV}) \times 10$ days. The solution of (27) approaches the limit $I_{\infty}=S / \lambda$ as $t \rightarrow \infty$. Suppose, however, that $I$ is reduced to zero by ralativistic electron precipitation (REP) events, which occur randomly in time. Thus, let $\exp (-\Delta t / \tau)$ be the probability that a time interval of length $\Delta t$ is free of $R E P$ events. The radiation intensity grows from zero in accordance with (27) after each REP event, and so never quite reaches $I_{\infty}$. A careful implementation of this model shows that the instantaneous probability for $I$ to be in excess of some arbitrary threshold $I_{0}<I_{\infty}$ at any given time is

$$
\mathrm{P}\left(\mathrm{I}>\mathrm{I}_{0}\right)=\left[1-\left(\mathrm{I}_{0} / \mathrm{I}_{\infty}\right)\right]^{1 / \lambda T}
$$

and that $\mathrm{P}\left(\mathrm{I}>\mathrm{I}_{\infty}\right)=0$.

Here is a simple proof of (28): If $t$ is the elapsed time following the most recent REP event, then is follows from (27) that

$$
I(t)=\left(1-e^{-\lambda t}\right)(S / \lambda)=\left(1-e^{-\lambda t}\right) I_{\infty}
$$

This is the solution of $(27)$ for $I(0)=0$. The intensity $I_{0}$ would thus be attained at time $t_{0}$, where

$$
\exp \left(-\lambda t_{0}\right)=\left[1-\left(I_{0} / I_{\infty}\right)\right]
$$

The probability that the immediately past interval of duration $t_{0}$ has been free of REP events is $\exp \left(-t_{0} / \tau\right)$. Thus, the probability that $I$ instantaneously exceeds $I_{0}$ is also exp $\left(-t_{0} / \tau\right)$. It follows from this consideration, and from $(30)$, that

$$
\begin{aligned}
P\left(I>I_{0}\right)= & \exp \left(-t_{0} / \tau\right) \equiv\left[\exp \left(-\lambda t_{0}\right)\right]^{1 / \lambda \tau} \\
& =\left[1-\left(I_{0} / I_{\infty}\right)\right]^{1 / \lambda \tau},
\end{aligned}
$$


as we have claimed in (28). Moreover, it follows from (29) that I will not exceed $\mathrm{I}_{\infty}$.

The mean value of $I$, averaged over a time interval of duration $\Delta t \gg \tau$, is given by

$$
\bar{I} \equiv \int_{0}^{I_{\infty}} I_{0}\left|d P / d I_{0}\right| d I_{0},
$$

since $\left|\mathrm{dP} / \mathrm{dI}_{0}\right| \mathrm{dI}_{0}$ is the probability of finding $\mathrm{I}$ between $\mathrm{I}_{0}$ and $\mathrm{I}_{0}+\mathrm{dI}_{0}$. In other words,

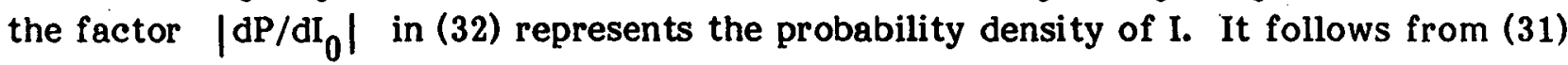
and (32) that

$$
\bar{I}=[\lambda \tau /(1+\lambda \tau)] I_{\infty}
$$

This is the natural state of affairs. The intensity of relativistic electrons in the vicinity of the plasmapause fluctuates in time in such a way that (28) and (29) are satisfied. Suppose, however, that REP events are suppressed, by the addition of heavy ions to the plasmasphere, causing suppression of ion cylotron waves. This environmental modification corresponds to the limit $\tau \rightarrow \infty$, whereupon $I=I_{\infty}$. The mean radiation intensity has been enhanced by a factor $(1+\lambda \tau) / \lambda \tau$. Thus, if $\tau=20$ days (corresponding to the annual occurrence of about 18 REP events), the intensity of $2-\mathrm{MeV}$ electrons will be enhanced by a factor $\sim 2$ (relative to its natural mean value) by the addition of major quantities of $\mathrm{Ar}^{+}$or $\mathrm{O}^{+}$to the plasmasphere. The mean intensity of 4-MeV electrons will be enhanced by a factor $\sim 3$. With the addition of heavy ions to the plasmasphere, the radiation intensity ceases to fluctuate between zero and $I_{\infty}$; it approaches $I_{\infty}$ as a permanent condition of the modified environment.

The above estimates of $I$ and $P\left(I>I_{0}\right)$ are based on a highly idealized model of radiation-belt dynamics. Other sources of fluctuation are quite likely to broaden the probability distribution. For example, one might expect both $\lambda$ and (especially) $S$ in (27) to depend on geomagnetic activity and therefore on time. Moreover, the magnetosphere is subject to reversible (adiabatic) compressions that change the scales of energy and distance. Such compressions (caused by slow variations of solar-wind speed) are not of dynamical interest and so are omitted from (27)-(29), but such adiabatic processes would have to be considered in formulating a model of the detailed time history of radiation dosage impacting a hardened spacecraft. 
The expected relativistic electron dosage increase estimated here is not sufficiently hazardous to preclude activity in space. However, increased dosage over long durations will undoubtedly impact space-borne equipment lifetimes. This is especially true for space systems operating in the regions of the radiation belts (earthward of GEO); an example among these is the Global Positioning System of twentyfour navigation satellites in $20,000 \mathrm{~km}$ orbits. Ironically, radiation belt modification is unlikely to impact SPS construction and operation at GEO if the modified plasmasphere does not extend further towards GEO. Nevertheless, it is rather likely that mitigation measures in the design of future space activities may have to be introduced to cope with radiation belt modification if SPS construction and operations become reality. This is especially true if the source of radiation belts are modified also.

\section{E. Plasma Instabilities and Effects on Space Communications}

As we have alluded to in previous Sections and Subsections, we expect the injection of the ion engine beam into the magnetosphere would cause turbulence in the form of plasma and electromagnetic waves. The effects on a particularly important mode, the electromagnetic ion cyclotron wave, have been dealt with in detail in Subsections V.C. and V. D. Thus, even the beam stopping process, discussed in Section IV, is not expected to be entirely a smooth deceleration by action of the Alfvén shocks alone, plasma turbulence stimulated by the plasma beam will undoubtedly play a contributory role. From a quasi-thermodynamic point of view, the beam-plasma system is highly unstable because the uniform streaming energy of the beam and the gradients of density and temperature set up between beam plasma and ambient magnetospheric plasma contain free energy. The evolution of this free energy into bound thermal energy is likely to take place by action of plasma instabilities which generate various wave modes in the plasma. In this Subsection, we shall first attempt to sort out the zoo of plasma instabilities which would be expected from SPS plasma injections. Some of these, such as the electromagnetic ion cyclotron instability, have been discussed previously; however, we have not yet discussed the effects of instabilities which generate plasma density irregularities which scatter communication signals. The second part of this Subsection shall be devoted to effects upon space communications.

The specific causes of plasma instabilities associated with argon plasma injection are a) relative streaming of one plasma (Ar) into another (ambient), b) velocity shear in the argon beam, c) spatial gradients of plasma parameters and d) residual 
Table III. Instabilities Relevant to SPS Plasma Injection

\begin{tabular}{|c|c|c|c|c|c|c|}
\hline Instability & $\begin{array}{c}\text { Character of } \\
\text { Wave }\end{array}$ & $\begin{array}{c}\text { Source of } \\
\text { Free Energy }\end{array}$ & $\begin{array}{c}\text { Special } \\
\text { Consideration }\end{array}$ & $\begin{array}{l}\text { Density } \\
\text { Irregularities }\end{array}$ & $\begin{array}{c}\text { Effect on } \\
\text { Argon Plasma }\end{array}$ & Alias \\
\hline $\begin{array}{l}\text { Modified } \\
\text { Beam Plasma }\end{array}$ & Electrostatic & $\begin{array}{l}\text { Relative } \\
\text { streaming in } \\
\text { velocity space } \\
\text { (Two-Stream) }\end{array}$ & Beam $\perp$ to $\vec{B}$ & Yes & Slows beam & $\begin{array}{l}\text { Cross-field } \\
\text { ion acoustic }\end{array}$ \\
\hline
\end{tabular}

Cross-field

Current-Driven

\section{Relative}

Electrostatic streaming in velocity space (Two-Stream)
Beam $\perp$ to $\vec{B}$

Yes

Slows beam

Farley-Buneman

Beam being bounded

in transverse

direction
Yes

\section{Disturbs beam}

$\begin{array}{ll}\text { Electrostatic } & \begin{array}{l}\text { Velocity } \\ \text { shear }\end{array}\end{array}$

Kelvin-Helmholtz

Drift Wave

\section{Electrostatic Spatial gradi-} ants of temperaants of tempera-
ture, density,
and composition ants of tempera-
ture, density,
and composition

\section{$\perp \vec{B}$}

\section{Ion temperature anisotropy}

Cyclotron

Harmonic

Electro-

Lef thanded

Ion temperature anisotropy magnetic

(EMC)

Electromagnetic
Electron
Cyclotron
(EMC)

$\begin{array}{ll}\text { Electro- } & \text { Temperature } \\ \text { magnetic } & \text { anisotropy of } \\ \text { Righthanded } & \text { hot electrons }\end{array}$

Electro-

Righthanded (EMC)
Enhanced by addition of cold plasma
After beam thermaliza- Yes tion
Yes Spreads beam
and plasma clo

Universal and plasma cloud instability
After beam thermalization
No

Pitch-angle diffusion
Ion Bernstein modes; Electrostatic Loss Cone 
temperature anisotropies left over after beam thermalization. A more comprehensive discussion of plasma instabilities in the magnetosphere can be found in Hasegawa (1975), although this reference does not specifically address SPS effects. The early consequence of such instability effects is the rapid amplification of thermal noise to appreciable amplitudes, such that the beam itself becomes diffused. Thus, plasma instabilities contribute to beam stopping; however, the question of how important these are in comparison to the action of Alfvén shocks is not yet known, since beam-plasma instabilities have not yet been investigated thoroughly in this context. A list of the major plasma instability species which are relevant to SPS plasma injection is given in Table III. This table is constructed in the hope of classifying and summarizing the characteristics and effects of the zoo of plasma instabilities which may be involved in the evolution of the argon beam free energy. The entries in the "Instability" column, running from top to bottom is meant to roughly parallel the sequence of this evolution. These involve not only the dense $\left(1-100 \mathrm{~cm}^{-3}\right)$, relatively cold $(\sim 1 \mathrm{eV})$ thermal plasma, but also ring-current and radiation-belt particles whose energies range from keV's to MeV's.

Another way of classifying the plasma instabilities may be by means of the time and spatial scales involved. This is especially relevant to the electrostatic modes which generate plasma density irregularities interferring with space communications. A partial list of processes in order of increasing time scale: cross-field current-driven instabilities (time scale $R_{e} / v \sim$ milliseconds, where $R_{e}$ is the electron Larmor radius); ion electrostatic modes (time scale $=\omega_{\mathrm{A}}^{-1}$, the $\mathrm{Ar}^{+}$plasma frequency $\approx 10^{-2} \mathrm{sec}^{-1}$ ); proton electromagnetic cyclotron (EMC) modes (time scale $\approx \Omega_{\mathrm{p}}^{-1} \approx 0.1 \mathrm{sec}$ ). These are important because they can cause local transfer of energy from the beam to the plasmasphere (first two modes), or because they affect natural magnetospheric processes. There are also magnetohydrodynamic instabilities of considerably larger time scale (e.g., Perkins et al., 1973), which cause deformations of the beam (striations, etc.), but which probably do not transfer much energy from beam to plasmasphere. KelvinHelmholtz and drift wave instabilities in Table III are some examples. The spatial scales (perpendicular to $\vec{B}$ ) are, as noted above, the electron and ion Larmor radius, probably with the argon ion Larmor radius as the outer spatial scale (see Table 1). Plasma density striations formed by the zoo of electrostatic modes cause signal scintillation effects which will be discussed below.

The anticpated signal scintillation effects, or equivalently the formation of plasma irregularities connected with ion engine beam-exhaust in the plasmasphere, can 
be attributed to various classes of electrostatic modes: a) the current-driven plasma instability of the exhaust beam such as entries 1 and 2 in Table III, and b) plasma drift instabilities associated with the density and velocity gradients of the exhaust plasma such as entries 3,4 and 5 in Table III. The former is very intense because the exhaust beam has a great deal of available free energy, but the turbulent region will probably be confined to the vicinity of the space transport. The latter includes a number of individual modes (typified by Kelvin-Helmholtz and drif $t$ modes) which may occur over an extensive region and at a range of plasmaspheric parameters since the instabilities are associated with the diffusion of the injected plasma cloud.

The cross-field current-driven ion acoustic instability has been observed in beam-plasma devices with operating conditions similar to those projected for ion engines (Barrett et al., 1972), except that the ion engines will have much higher beam current. This instability draws its energy from the free energy of the streaming plasma beam and generates ion acoustic waves (density irregularities) propagating at large angles to the magnetic field. These waves have frequencies well below the electron cyclotron frequency and have wavelengths of the order of the electron gyroradius, which is $\sim 30 \mathrm{~cm}$ in the ionosphere and is $\sim 20 \mathrm{~m}$ at $4 \mathrm{R}_{\mathrm{E}}$. Since signal scintillation effects are most severe when the signal wavelength closely matches the irregularity size, we expect that the $30 \mathrm{~cm}$ irregularities of the beam-plasma instability will cause signal scintillation effects at carrier frequencies of $\sim 1 \mathrm{GHz}$. Currently, most civilian and military communication and navigation systems operate in this frequency region.

Drift instabilities draw their energies from the free energy available in plasma density gradients and are instrumental in the diffusion of plasma density concentrations. Observational evidences of drift-induced density striations are available from numerous plasma injections in the magnetosphere ranging from barium releases to nuclear detonations at high altitude. Since these instabilities are not directly related to the beam-exhaust, but are related to the plasma cloud af ter the streaming plasma beam motion has been randomized, we would expect that rather large areas will be striated. This has recently been confirmed by the CAMEO barium release experiment (Smith et al., 1979). Further, since there is a virtual zoo of drift related instabilities, the scale sizes of the density irregularities will probably be broadband; although, if natural equatorial spread-F condition is any guide, drift-induced density irregularities will probably affect VHF and UHF more severely than frequencies in the gigahertz range. 
In summary, our assessment effort has identified that plasma instabilities will likely occur and play a significant role in the energy evolution of SPS plasma injections by the COTV. The electrostatic modes of these plasma instabilities generate density irregularities in the form of plasma striations aligned with the geomagnetic field. These irregularities are of the proper size to be potential scattering centers for satellite communication signals, causing signal scintillation effects. The strength of communication interference effects cannot be determined in the present assessment effort because this will require more complete research work. In the natural case, signal scintillation effects are primarily caused by plasma irregularities below $1000 \mathrm{~km}$ altitude because magnetospheric irregularities are not strong enough to produce significant effects. Booker (1975), however, pointed out that signal interference effects require spatial integration over the extent of the scatterers and that even weak irregularities may produce signal interference if they are extensive enough. Recently, the CAMEO barium plasma release experiment at $\sim 500 \mathrm{~km}$ altitude (Smith et al., 1979) showed density striations extending to $\gtrsim 10,000 \mathrm{~km}$ altitude. Obviously, this area is still under active scientific investigation. We cannot yet determine with some degree of certainty the severity of potential communication signal interference effects of SPS plasma injections, although the dynamical role of plasma instabilities in magnetospheric modifications is surely to be very significant. 


\section{THIS PAGE \\ WAS INTENTIONALLY \\ LEFT BLANK}

$=$

$\because$ 
VI. EFFECTS OF NEUTRAL EXHAUST (POTV)

Assessment of POTV neutral exhaust effects in the magnetosphere is not originally called for in FY79 contract activities. However, in the course of assessing SPS effects on modification of ring-current and auroral processes (including modification of induced ionospheric currents which may cause power-line surges), we came to realize that the quantity of neutral exhaust and the location of its injection is so critical to ring current processes that assessment of auroral process modification would be quite meaningless unless the effects of the neutrals were included. For this reason, we include here our initial assessment of POTV exhaust effects. Our discussions will be brief and qualitative: In the nature of leading into the projected assessment program in FY80 rather than in the nature of presenting assessment results for FY79.

The main effect of the neutral exhaust cloud is that it acts as an artificial source that can charge-exchange with ring-current ions, thereby reducing the lifetime of these energetic particles and modifying auroral activity. Natural charge-exchange lifetimes for ring current ions $\mathrm{E} \sim 1-100 \mathrm{keV}$ are $\sim 10^{5} \mathrm{sec}$, as can be seen from the duration of the recovery phase of a geomagnetic storm. If the ring-current particle lifetimes are significantly shortened by charge exchange interaction with the neutral cloud, auroral activity would be significantly reduced by the following sequence of effects: (a) chargeexchange reaction converts an energetic charged particle into an energetic neutral which immediately escapes geomagnetic confinement; (b) ring current precipitation and trapped fluxes are reduced, leading to (c) reduction of strength and duration of geomagnetic storms and ionospheric disturbances. Taken from a narrow viewpoint, if geomagnetic disturbances can be considered as interfering with the smooth functioning of a number of technological activities, this potential magnetospheric effect may be considered beneficial. However, it must be noted that it would be premature at present to assess such effects as being of benefit or harm.

To estimate the reduction of ring current lifetimes by charge exchange, we make use of (1) in Section II: $\tau=1 / N_{n} \sigma v$, where $N_{n}$ is the mean neutral density on the drift shell of the ring-current ion. The neutral exhaust is initially $\mathrm{H}_{2} \mathrm{O}$, which may undergo various charge-exchange reactions resulting in $\mathrm{H}_{2} \mathrm{O}^{+}$as in the case of "ionospheric holes" or photo-dissociate into neutral atomic constituents. In any case, it is quite clear that $\mathrm{N}_{n}$ and $\sigma$ above will involve various species of neutral atoms or molecules, requiring a careful assessment of chemical processes. However, the upper limit of $\tau$ can be 
evaluated by assuming the photodissociation case because charge-exchange crosssections for molecules are necessarily larger than atomic charge-exchange cross sections. Further, since we are only interested in order-of-magnitude estimates, we shall limit ourselves to charge exchange of the ring-current ions with neutral hydrogen atoms. The quantities $\sigma \mathrm{v}$ for neutral-hydrogen charge exchange with ring-current particles of various energies and species are quite well known. These are shown on Figure 12, taken from Cornwall and Schulz (1979). The primary natural ring-current species are $\mathrm{H}^{+}, \mathrm{He}^{+}, \mathrm{He}^{++}$and $\mathrm{O}^{+}$; under conditions of SPS modification, $\mathrm{Ar}^{+}$may also be added. As is discussed in Section II, an order-of-magnitude estimate of the lifetimes of various ring-current ions mirroring in the equatorial plane may be obtained by using Figure 12 and a model of neutral-cloud expansion such as that discussed in Subsection V.A. and Section III. Evaluation of the drift-shell-averaged density $N_{n}$ for neutral jet release at $R=5 R_{E}$ yields $N_{n} \sim 10^{4}$ atoms $/ \mathrm{cm}^{3}$. Thus, from Figure 12 , we determine that $10-\mathrm{keV}$ ring-current protons would have a lifetime of $\sim 1000 \mathrm{sec}$ and a $10-\mathrm{keV} \mathrm{O}^{+}$would have a lifetime of $\sim 2 \times 10^{4} \mathrm{sec}$ at $5 \mathrm{R}_{\mathrm{E}^{*}}$. Compared to natural ring-current lifetimes of $\sim$ $10^{5} \mathrm{sec}$, we see that the effects of neutral exhaust upon ring-current dynamics may be quite drastic.

Radiation-belt particles have much higher energies $(E \gtrsim 100 \mathrm{keV}$ ), and so, according to Figure 12, they are relatively immune to charge-exchange loss induced by the neutral exhaust cloud. However, a plasma effect (not yet understood theoretically) may take place and convert a fraction of the neutrals into thermal ions, in which case the processes discussed in Subsections V.C. and V.D. concerning suppression of electromagnetic cyclotron instability will become operative if the ion is $\mathrm{O}^{+}$. This plasma effect is the critical-velocity phenomenon (Alfvén and Arrhenius, 1976; Angerth et al., 1962; Danielsson and Brenning, 1975; Möbius et al., 1979) which suggests that a neutralparticle cloud moving with velocity relative to the ambient plasma greater than $\mathbf{v}_{\mathbf{c}}$ $\equiv\left(\text { le } \mid V_{i} / m\right)^{1 / 2}$ is apt to be ionized, where $|e| V_{i}$ is the ionization potential energy of the neutral particles of mass $\mathrm{m}$. Although the Alfvén critical-velocity phenomenon needs to be verified further in the laboratory and in space, some preliminary evidence for it in space may have been observed in the interactions between a lunar dust cloud and the solar wind (Lindeman et al., 1974). Since $v_{c}$ is $\sim 50 \mathrm{~km} / \mathrm{sec}$ for hydrogen and $\sim 13$ $\mathrm{km} / \mathrm{sec}$ for oxygen, it is possible that the critical-velocity phenomenon may produce a large source of cold $\mathrm{O}^{+}$ions, especially, since rocket speeds of $\sim 13 \mathrm{~km} / \mathrm{sec}$ are achieved during the passage from LEO and GEO. The effects of this extra source of thermal $\mathrm{O}^{+}$ 


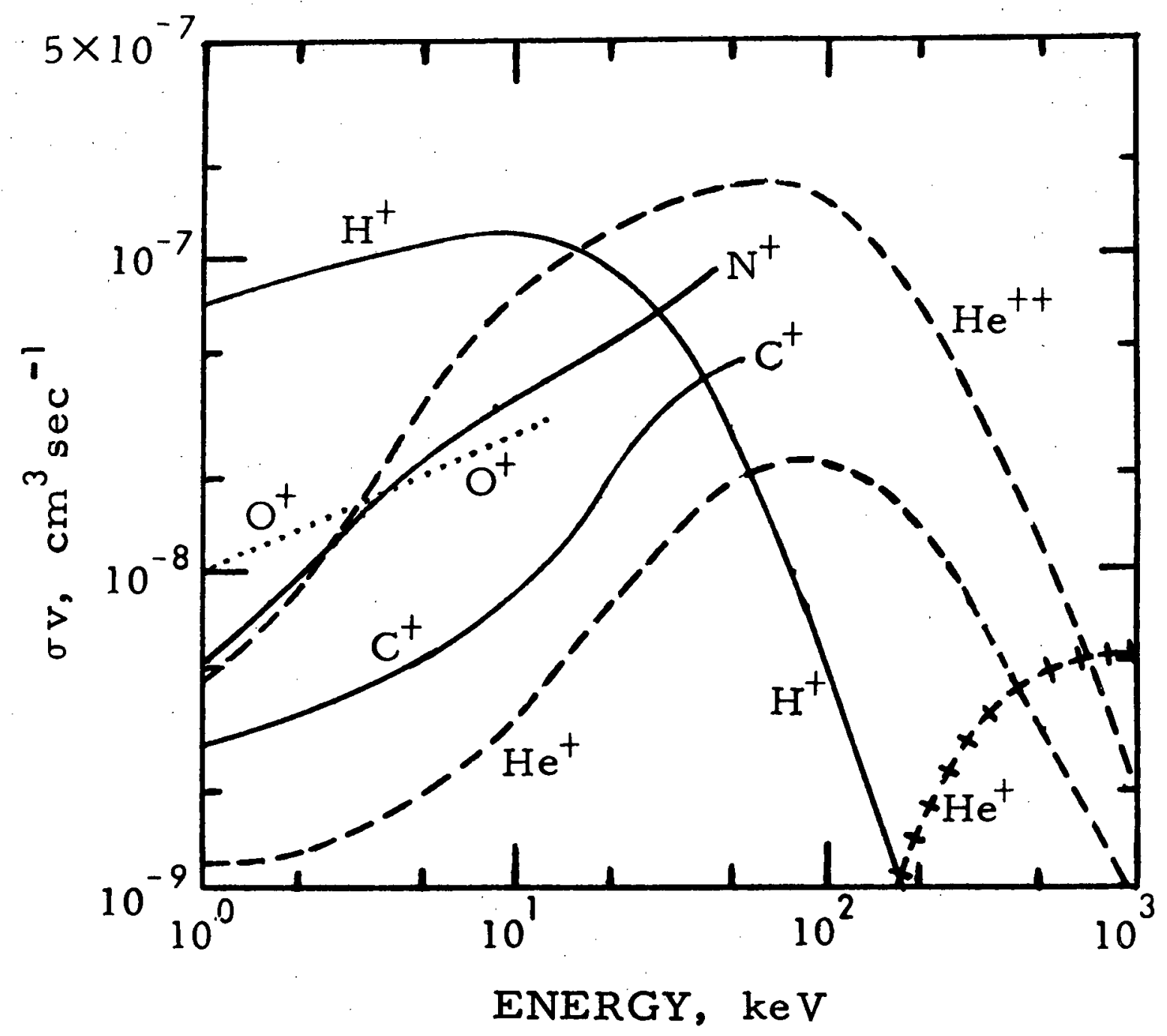

Figure 12. Product $(\sigma \mathrm{v})$ of Charge-Exchange Cross Section $\sigma$ (between Indicated Ions and Neutral Hydrogen Atoms) and Ion Speed $v$ Shown as Functions of Ion Energy. One of the curves for $\mathrm{H}^{+}$(composed of crosses) is for stripping while the other is for charge exchange. 
ions in the plasmasphere would be the same as the effects that have been described previously: suppression of ring current ion-cyclotron instability and enhancement of radiation-belt relativistic-electron intensity (see Subsections V.C. and V.D.). Therefore, if the critical-velocity phenomenon observed in the laboratory, is applicable in space, the operation of chemical rockets in the magnetosphere will generate ion clouds which behave in much the same manner as the argon-ion clouds.

In summary, the large amount of POTV neutral exhaust has drastic effects on the ring current because ring-current particles rapidly charge-exchange with the neutral cloud and escape magnetic confinement. The result is likely to be a shortened duration and reduced intensity of auroral geomagnetic storms. In addition, neutral $O$ atoms from the chemical exhaust may constitute an extra source of thermal $\mathrm{O}^{+}$ions in the plasmasphere, giving rise to enhancement of relativistic electron flux in the radiation belts. It appears that evaluation and assessment of this new source of magnetospheric effects must be evaluated in FY80 before a more complete and integrated assessment of SPS magnetospheric effects can be made. 


\section{SUMMARY OF MAGNETOSPHERIC EFFECTS}

Exhaust emissions from propulsion and stationkeeping activities of SPS spacecraft induce substantial modifications of magnetospheric processes on both the local and the global scale. This is primarily because of the relatively large mass and energy contents of these emissions when compared with the total mass and energy contents of the inner magnetosphere. The sources of these emissions are: (a) the argon plasma jet from the solar electric propulsion modules of the cargo orbit transfer vehicle (COTV), and (b) the $\mathrm{H}_{2} \mathrm{O}$ neutral exhaust from $\mathrm{LO}_{2} / \mathrm{LH}_{2}$ main engines of the personnel orbit transfer vehicles (POTV). Assessment of the SPS scenario, based on presently-known physical mechanisms operative in plasma and neutral injections in the magnetosphere, indicates that the major part of the exhaust emissions are likely to be deposited inside the magnetosphere.

Injection of argon ions of $3.5 \mathrm{keV}$ energy causes magnetospheric modification in several ways. The energy released is sufficient to cause turbulent response in the magnetospheric plasma. As this free energy evolves, the magnetospheric composition is modified not only by the presence of argon ions but also by the heating. This physical evolution of injected energy and mass causes an increase in the intensity of radiation belt relativistic electrons which may require mitigation on the part of systems design in space equipment and human activity. At low earth orbit (LEO), a substantial fraction of the energetic argon may escape magnetic confinement and impact the atmosphere in the form of an intense beam. The optical emissions stimulated by such a beam may be more than an order of magnitude more intense than the aurora at near UV wavelengths. The possible interference with space-borne optical sensors induced by such a strong source of artificial atmospheric emissions may require further technological assessment by the sensor community. The earth's response to solar disturbances, in the form of auroral magnetic storms, depends on the density and composition of the magnetospheric constituents (plasmas and neutrals); modification of the magnetospheric density and composition is likely to change the magnetospheric response to solar activity. Because of the rapid rate of charge exchange interaction between energetic particles of solar wind origin and the neutral exhaust cloud from the POTV, we expect that the earth's response to solar activity may become shorter in duration and weaker in intensity under SPS-modified circumstances. Further, some plasma disturbances caused by the transformation of injected free energy involve density irregularities and currents to form in 
the magnetospheric and ionospheric plasma. If these density irregularities are of sufficiently large extent, they may cause signal scintillation effects in space communication systems. The ionospheric currents induced in the ionosphere are of comparable magnitude to the auroral currents but are located at mid-latitudes; thus they may adversely impact powerlines and pipelines.

For the benefit of the reader, Table IV lists the salient features of the principal SPS magnetospheric effects discussed above. The listing is not ordered according to importance of impact, because we deem it premature to make such judgments at the current stage of assessment. However, some general judgmental impressions can be made at the present stage if such are called for. It appears to us that the principal magnetospheric effects are sufficiently serious to require mitigation measures on the part of space systems design. Indeed, radiation belt modifications may impact upon the SPS system design itself. However, it is also our general impression at this point that these effects are not sufficiently serious to put a stop to the project. 
Table IV. Satellite Power System Magnetospheric Effects

\begin{tabular}{llcc}
\hline Effect & Cause & Mechanism & System/Activities \\
Impacted
\end{tabular}

1. Dosage Enhancement of Trapped Relativistic Electrons

\section{Artificial}

$\mathrm{O}^{+}$and $\mathrm{Ar}^{+}$in magnetosphere due to exhaust and plasmasphere heating

Ionospheric electric

field induced by argon beam

Neutrals and heavy ions in large quantities Response to Solar Activity

\section{Artificial Airglow}

5. Plasma Density Disturbance on Small Spatial Scale
Thermal heavy ions suppress ring - Space equipment current ion cyclotron turbulence, - Modification of human space which keeps electron dosage in activity balance in natural state

Beam induced Alfvén shocks propagate into ionosphere

- Powerline tripping

- Pipeline corrosion

Rapid charge-exchange loss of ring-current particles

- May reduce magnetic storm interference with earth and space-based systems

Direct impact on atmosphere

- Interference with optical from LEO source earth sensors

Plasma instabilities

- Signal scintillation for spacebased communication 


\section{THIS PAGE \\ WAS INTENTIONALLY \\ LEFT BLANK}

$z^{\prime}$

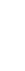


VIII. RECOMMENDATIONS FOR FINAL ASSESSMENT

Even though the foregoing report deals only with the results of an initial assessment of magnetospheric effects of the SPS, a number of general and specific impressions have been gained in the course of studying the physical implications of the principal issues treated in the previous Section. These impressions may be germane to the development of a final assessment program. Indeed, some specific impressions may be critical elements in determining the direction of future efforts in the project, not necessarily with the specificity of an environmental impact statement but with a view towards establishing a physically sound basis to define the approximate limits of magnetospheric modification. These impressions are set forth below:

1. In order to deal with the physics of magnetospheric modification, it is of critical importance to know unequivocally the quantity and characteristics of exhaust emissions in space and time. We have not been able to find such a collection of SPS parameters defined primarily for environmental assessment. Emission parameters for COTV and POTV engines used in this report are gleaned from brief entries in the concept definition report (U.S. DOE, 1979) and the SPS Baseline Review (U.S. NASA, 1978) which deal primarily with engineering development. Since some principal magnetospheric effects such as radiation belt dosage enhancement may impact upon the engineering design of SPS itself, it would be advantageous to have a definitive compilation of SPS emission and other parameters of concern to environmental assessment.

2. Magnetospheric modification by plasma beam injection is a topic of current scientific interest; consequently, the results of these current researches can be applied to SPS magnetospheric effects (the present effort being one of such). However, the scale of SPS magnetospheric modifications is so many orders of magnitude larger than present modification experiments that theoretical efforts must be applied to determine the probable effects. In this initial assessment the emphasis is on examination of the physical basis of magnetospheric effects and selection of principal areas of concern. We recommend that the second stage of semi-realistic scaling-up of the principal magnetospheric issues to SPS dimension be attempted before the FY 80 final assessment. More specifically, effects such as determining the severity (if any) of interference with powerline and pipeline operations by ionospheric currents (artificially induced by the SPS plasma beam) can be evaluated; but to do so, we must first perform in-depth modeling of the Alfvén shock electric field propagation in a semi-realistic 
plasmasphere. Without such semi-original applied research, the assessment results would remain indeterminate.

3. Data on physical processes relevant to SPS assessment, such as argon ion charge-exchange and photo-excitation cross-sections, are generally considered to be exotic and not relevant to natural atmospheric physics. Consequently, there has been little research on such processes; and, the results of whatever research that is relevant to SPS are usually archived in out-of-the-main stream journals. A semi-empirical research effort on atmospheric chemistry may benefit not only magnetospheric effects assessment but also assessment for ionospheric and upper atmospheric regions as well.

4. Because of the paucity of physical data and the lack of a definitive compilation of emission rates and characteristics, comprehensive environmental modeling on physically uncertain (or not yet fully understood) issues, such as possible connections between magnetospheric effects and lower atmospheric climate, do not seem to be cost-effective. However, specific physical modeling on principal issues are absolutely essential for final assessment.

5. It appears to us at the present stage that the five principal effects listed in Table IV are of more immediate concern in the magnetospheric effects area. We recommend in-depth study and scaling (up to SPS dimensions) for these issues. Note that some of these issues are caused by a complex chain of events of ten involving both argon ion engine and chemical engine exhausts.

6. On a longer term viewpoint, observational verification of the assessment is absolutely necessary; therefore, we recommend coordination and support of experimental programs on magnetospheric modification.

7. Although we have attempted to assess in the short time available as many magnetospheric effects as we have considered to be of importance, we realize that important issues will probably be discovered in the future. The POTV neutral exhaust issue is a very good example. Aside from this, a couple of important issues have been recognized by us but are not reported here because assessment and coordination are continuing. First, the enhanced loss of hydrogen from POTV neutral exhaust released in the magnetosphere is not only a substantially larger loss of hydrogen from the earth, but is also a source of increased geocorona which may impact astronomy. Further, D-region ionospheric electron density is critically dependent on Lyman- $\alpha$ emission intensity from the geocorona. This effect will be investigated in FY 80. Second, we have coordinated with observations of spacecraft charging effects by the SCATHA satellite and we expect 
to report on its assessment more fully in FY 80 when data from SCATHA will be more fully analyzed. 
THIS PAGE

\section{WAS INTENTIONALLY \\ LEFT BLANK}




\section{REFERENCES}

Alfvén, H., and G. Arrhenius, Evolution of the Solar System, National Aeronautics and Space Administration NASA SP-345, U. S. Gov't Printing Office, Washington D. C., Ch. 21; pp 383-391, 1976.

Angerth, B., L. Block, U. Fahlson, and K. Sopp, Experiments with partly ionized rotating plasmas, Nucl. Fusion Supp. Part I, 39, 1962.

Baker, D. J., and W. R. Pendleton, Jr., Methods for atmospheric radiometry, Proceedings of Society of Photo-Optical Instrument Engineers, D. P. MeNutt (editor), SPIE, 91, 1976.

Barrett, P. J., B. D. Fried, C. F. Kennel, J. M. Sellen and R. J. Taylor, Cross-field current-driven ion acoustic instability, Phys. ㄹev. Letters, 28, 337, 1972.

Booker, H. G., The role of the magnetosphere in satellite and radio-star scintillation, Effects of the Ionosphere on Space Systems and Communications, J. M. Goodman (editor), Naval Research Laboratory, pp. 9-12, 1975.

Brice, N., Artificial enhancement of energetic particle precipitation through cold plasma injection: A technique for seeding substorms, J.Geophys. Res., 75, 4890, 1970.

Brice, N., Harnessing the energy in the radiation belts, J. Geophys. Res., 76, 4698, 1971.

Byers, D. C. and V. K. Rawlin, Electron bombardment propulsion system characteristics for large space systems, Twelfth International Electric Propulsion Conference, American Institute of Aeronauties and Astronautics, Key Biscayne, Fla., Nov. 15-17, 1976.

Ching, B. K., and Y. T. Chiu, Global distribution of thermospheric heat sources: EUV absorption and Joule dissipation, Planet. Space Sci., 21, 1633, 1973.

Chiu, Y. T., J. G. Luhmann, B. K. Ching, Michael Schulz and D. J. Boucher, Jr., Magnetospheric and ionospheric impact of large-scale space transportation with ion engines, Aeronautics and Astronautics, accepted for publication, 1979a; also Aerospace Technical Report SAMSO-TR-79-3, 1979a.

Chiu, Y. T., L. G. Luhmann, B. K. Ching and D. J. Boucher, Jr., An equilibrium model of plasmaspheric composition and density, J. Geophys. Res., 84, 909, $1979 \mathrm{~b}$. 
Chiu, Y. T., J. M. Cornwall, J. G. Luhmann and M. Schulz, Argon-ion contamination of the plasmasphere, Progress in Astronautics and Aeronautics (series editor: M. Summerfield): Space Systems and their Interactions with the Earth's Space Environment" (Vol. editors: H. B. Garrett and C. P. Pike), AIAA, New York, 1979c.

Cornwall, J. M., Micropulsations and the outer radiation zone, J. Geophys. Res., 71, $2185,1966$.

Cornwall, J. M., On the role of charge exchange in generating unstable waves in the ring current, J. Geophys. Res., 82, 1188, 1977.

Cornwall, J. M., F. V. Coroniti, and R. M. Thorne, Turbulent loss of ring current protons, J. Geophys. Res., 75, 4699, 1977.

Cornwall, J. M., and M. Schulz, Electromagnetic ion-cyclotron instabilities in multicomponent magnetospheric plasmas, J. Geophys. Res., 76, 7791, 1971; Correction, J. Geophys. Res., 78, 6830, 1973.

Cornwall, J. M., and M. Schulz, Physics of heavy ions in the magnetosphere, in Solar System Plasma Physics, Vol. III, Ch. III.1.5, editors: L. J. Lanzerotti, C. F. Kennel and E. N. Parker, North-Holland, Amsterdam, 1979.

Curtis, S. A., and J. M. Grebowsky, Changes in the terrestrial atmosphere-ionospheremagnetosphere system due to ion propulsion for solar power satellite placement, NASA Tech. Memo. 79719, Goddard Space Flight Center, unpublished, 1979.

Danielsson, L., and N. Brenning, Experiment on the interaction between a plasma and a neutral gas, II, Phys. Fluids, $18,661,1975$.

Drell, S. D., H. M. Foley, and M. A. Ruderman, Drag and propulsion of large satellites in the ionosphere: an Alfven propulsion engine in space, J. Geophys. Res., 70, 3131, 1965.

Gilbody, H. B., and J. B. Hasted, Anomalies in adiabatic interpretation of charge transfer collisions, Proc. Roy. Soc., Ser. A, 238, 1956.

Haerendel, G., and R. LUst, Electric fields in the ionosphere and magnetosphere, Particles and Fields in the Magnetosphere (ed. B. M. McCormac), p. 212, Reidel, Dordrecht-Holland, 1970.

Hasegawa, A., Plasma Instabilities and Nonlinear Effects, pp. 28-43, Springer, Heidelberg, 1975.

Hedrick, A. F., T. F. Moran, K. J. McCann, and M. R. Flannery, Charge transfer cross sections in argon ion-diatomic molecule collisions, J. Chem. Phys., $\underline{66}, 24,1977$. 
Kauffman, H. R., Technology of electron-bombardment ion thrusters, Advances in Electronics and Electron Physics, Vol. 36, ed. L. Marton, Academic Press, New York, 1974.

Kennel, C. F., and H. E. Petschek, Limit on stably trapped particle fluxes, J. Geophys. Res., $\underline{71}, 1,1966$.

Lindeman, R. A., R. R. Vondrak and J. W. Freeman, The interaction between an impactproduced neutral gas cloud and the solar wind at the lunar surface, J. Geophys. Res., $\underline{79}, 2287,1974$.

Liu, C. and H. P. Broida, Optical spectra observed during ion-molecule collisions using low-energy $\mathrm{N}_{2}^{+}$and $\mathrm{Ar}^{+}$beams, Phys. Rev., 2A, 1824, 1970.

Lyons, L. R., and D. S. Evans, The inconsistency between proton charge exchange and the observed ring current decay, J. Geophys. Res., 81, 6197, 1976.

Märk, E., Growth rates of ion cyclotron instability in the magnetosphere, J. Geophys. Res., 79, 3218, 1974.

Mobbius, E., R. W. Boswell, A. Piel, and D. Henry, Investigation of the critical velocity phenomenon from SPACELAB, Transact. AGU/EOS, 59, 1162, 1979.

Perkins, F. W., N. J. Zabusky, and J. H. Doles, III, Deformation and Striation of plasma clouds in the ionosphere, 1, J. Geophys. Res., 78, 697, 1973.

Pilipp, W. G., Expansion of an ion cloud in the earth's magnetic field, Planet. Space Sci., $19,1095,1971$.

Scholer, M., On the motion of artificial ion clouds in the magnetosphere, Planet. Space Sci., 18, 977, 1970.

Schulz, M., Particle saturation of the outer zone: A nonlinear model, Astrophys. Space Sci., 29, 233, 1974.

Schulz, M., and L. J. Lanzerotti, Particle Diffusion in the Radiation Belts, Springer Verlag; Berlin, 1973.

Smith, G. M., M. B. Pongratz, H. Fishbine, L. B. Davelsberg, and J. P. Heppner, Triangulation measurements of the acceleration of CAMEO barium ions parallel to the geomagnetic field, Trans. AGU/EOS, 60, 351, 1979. 
Tinsley, B. A., Evidence that the recovery phase ring current consists of helium ions, J. Geophys. Res., 81, 6193, 1976.

Thomas, E. W., Excitation in Heavy Particle Collisions, Wiley-Interscience, New York, 1972.

Thorne, R. M., and C. F. Kennel, Relativistic electron precipitation during magnetic storm main phase, J. Geophys. Res., 76, 4446, 1971.

U. S. Department of Energy, Satellite power system concept development and evaluation program, DOE/ER-0023, 1979.

U. S. National Aeronautics and Space Administration, Solar power satellite baseline review, Marshall Space Flight Center and Johnson Space Flight Center, 1978.

U. S. National Oceanic and Atmospheric Administration, Dept. of Commerce, Solar Terrestrial Predictions Proceedings, editor: R. F. Donnelly, Vol. I, Boulder, Colo., 1980 .

Vampola, A. L., Electron pitch angle scattering in the outer zone during magnetically disturbed times, J. Geophys. Res., 76, 4685, 1971.

White, R. S., The earth's radiation belts, Phys. Today, $19(10), 25$, October 1966.

Zinn, J., H. Hoerlin, and A. G. Petschek, The motion of bomb debris following the Starfish test, Radiation Träpped in the Earth's Magnetic Field (Ed. B. M. McCormac), Reidel, Dordrecht, Holland. 